\title{
Exploiting Backscatter-Aided Relay Communications with Hybrid Access Model in Device-to-Device Networks
}

\author{
Shimin Gong, Lin Gao, Jing Xu, Yuanxiong Guo, Dinh Thai Hoang, and Dusit Niyato
}

\begin{abstract}
The backscatter and active RF radios can complement each other and bring potential performance gain. In this paper, we envision a dual-mode radio structure that allows each device to make smart decisions on mode switch between backscatter communications (i.e., the passive mode) or RF communications (i.e., the active mode), according to the channel and energy conditions. The flexibility in mode switching also makes it more complicated for transmission control and network optimization. To exploit the radio diversity gain, we consider a wireless powered device-to-device network of hybrid radios and propose a sum throughput maximization by jointly optimizing energy beamforming and transmission scheduling in two radio modes. We further exploit the user cooperation gain by allowing the passive radios to relay for the active radios. As such, the sum throughput maximization is reformulated into a non-convex. We first present a sub-optimal algorithm based on successive convex approximation, which optimizes the relays' reflection coefficients by iteratively solving semi-definite programs. We also devise a set of heuristic algorithms with reduced computational complexity, which are shown to significantly improve the sum throughput and amenable for practical implementation.
\end{abstract}

Index Terms-Hybrid radio, wireless backscatter, wireless power transfer, beamforming, relay communications

\section{INTRODUCTION}

Recently, wireless backscatter has been proposed to enable direct communications between user devices by leveraging the ambient RF signals in the environment [1]. The backscatter radios transmit information in the passive mode by reflecting and modulating bit stream on the incident RF signals, whereas the conventional radios operate in the active mode relying on self-generated carrier signals. The information modulation of passive radios is performed by adapting the load impedance and correspondingly changing the antennas' reflection coefficients. Without using power-consuming components, e.g., analog/digital converter, crystal oscillator, and power amplifier, the passive radios consume orders of magnitude less power than the active radios, making it possible for RF-based wireless power transfer to sustain low-power wireless backscatter

Shimin Gong is with the School of Intelligent Systems Engineering, Sun Yat-sen University, China, email: gong0012@e.ntu.edu.sg; Jing Xu is with the School of Electronics Information and Communications, Huazhong University of Science and Technology, China, email: xujing@hust.edu.cn; Lin Gao is with the Department of Electronic and Information Engineering, Harbin Institute of Technology (Shenzhen), China, email: gaolin021@hotmail.com; Yuanxiong Guo is with the School of Electrical and Computer Engineering, Oklahoma State University, USA, email: guoyuanxiong@gmail.com; Dinh Thai Hoang is with the School of Electrical and Data Engineering, University of Technology Sydney, Australia, email: hoang.dinh@uts.edu.au. Dusit Niyato is with the School of Computer Science and Engineering, Nanyang Technological University, Singapore. Email: dniyato@ntu.edu.sg. communications for billions of user devices constituting the Internet of Things (IoT) [2].

\section{A. Motivation}

The passive radios rely on the RF signals emitted from the active RF communications. The operation of passive radios may also affect the channel conditions and thus the transmission performance of the active radios, e.g., [3] and [4]. Such dependence can be exploited to improve the overall performance of a hybrid radio network consisting of both the passive and active radios. On the other hand, the integration of two radio modes also complicates the performance maximization of the hybrid radio network. The passive radios use the RF signals to instantly reflect back the information bits, while the active radios can harvest, store, and use the RF energy for RF communications. Such difference requires a joint system design to maximize the sum throughput performance.

In this paper, we consider a device-to-device (D2D) network consisting of multiple hybrid radios, RF-powered by a multi-antenna power beacon station (PBS). Each D2D user equipment (DUE) has a dual-mode transceiver structure that can switch between two radio modes by controlling the load impedance. Our objective is to maximize the sum throughput by allowing each user to optimize its radio mode and the cooperative transmission strategy. Specifically, our design problem mainly concerns two aspects to improve the overall network performance. Firstly, we exploit the radio's diversity gain by allowing radio mode switching. The user device can flexibly schedule its data transmissions in two different modes based on its energy status and channel conditions. Secondly, we exploit the cooperative gain by allowing the passive radios to serve as wireless relays for the active RF communications. Instead of decoding and forwarding, each passive relay can set a fixed reflection coefficient to instantly reflect the incident RF signals from the source node to its receiver, mimicking the full-duplex relaying communications. By choosing a proper reflection coefficient, the backscattered signals can be leveraged as a form of multi-path diversity gain to enhance the signal reception at the active receiver [5].

\section{B. Contributions}

Our contributions in this paper are two-fold. Firstly, we study the throughput maximization problem in two cases to exploit performance gain from radio diversity and user cooperation, respectively. For the non-cooperative case, the DUEs are 
independent and scheduled to transmit data in a time-slotted frame structure. We formulate a convex throughput maximization problem to optimize the PBS' energy beamforming and the DUEs' transmission scheduling strategies. By timeswitching scheduling, we can avoid interference in concurrent RF communications among different users. However, individual users are still strongly coupled by their energy budget constraints. The PBS can adjust its energy beamforming strategies in different time slots, which will affect the energy supply of different users and their optimal working modes. We further consider the cooperative case, in which we employ the passive radios as wireless relays for active communications. This implies that the passive and active radios are complement in data transmissions, and thus a joint optimization of all radios' working modes is required to maximize the overall network performance. Due to users' coupling in mode switching and relaying communications, the throughput optimization is very challenging and untouched before.

The other contribution lies in that we devise both approximate and simple heuristic algorithms to solve the non-convex throughput maximization problem. Based on successive convex approximation (SCA), the approximate algorithm returns a sub-optimal solution by iteratively solving semi-definite programs. A set of heuristic algorithms are also proposed to further reduce the computational complexity. The performance comparison of different heuristic algorithms is partly evaluated in [6]. The simplest passive relaying scheme is to enforce all passive radios to relay for the active transmissions, which is denoted as the myopic relay cooperation (MRC) scheme. It achieves near-optimal performance when the DUEs' energy supply is insufficient while its performance becomes worse off with sufficient energy supply. We also propose the Equal Residual Energy (ERE) scheme that allows each passive relay to intercept a fixed amount of RF power from each transmitter in active mode. While the other part of the incident RF power will be reflected back to enhance signal reception. Both relay schemes can significantly improve the sum throughput compared to the non-cooperative case without the passive relaying communications. The simulation results also reveal that the ERE scheme performs practically good in terms of performance, overhead, and computational complexity, compared to a greedy algorithm as well as the SCA-based approximation.

The rest of this paper is organized as follows. We present an overview on related works in Section II and propose the hybrid radio network in Section III. Then, we formulate the throughput maximization problem and optimize the binary radio mode selection in Section IV. Furthermore, we reformulate the optimization problem to account for the passive relaying communications in Section V, which is solved by both approximate and heuristic algorithms. Evaluations and conclusions are given in Sections VI and VII, respectively.

\section{RELATED WORKS}

Wireless backscatter typically has three different configurations. It is conventionally used by radio frequency identification (RFID), where a high power reader excites and retrieves information from nearby passive tags [7]. This is referred to as the monostatic configuration with co-located receiver and RF emitter on the same device, e.g., the RFID reader. The newly developed bistatic configuration separates the RF emitter from the receiver [1]. It unleashes the passive tags from the bulky reader and thus provides better mobility for the passive radios. In this case, the backscatter receiver can be configured with the knowledge of RF signals to achieve much higher data rate, e.g., [8] and [9]. Wireless backscatter can also appear in the ambient configuration, which supports direct communications between two peer passive radios by leveraging ambient RF signals emitted from existing devices, e.g., TV towers [10], [11], cellular base stations [12], [13], and Wi-Fi devices [8], [14]. As the ambient RF signals are stochastic in nature and unpredictable, different signal detection and encoding mechanisms have been proposed to improve the performance of ambient backscatter communications, e.g., [15] and [16].

By allowing radio mode switching, the user device can flexibly schedule its data transmissions in two modes based on its energy status and channel conditions. For example, the user device can operate in the passive mode when the channel is occupied and switch to the active mode when the channel is idle. This case can be regarded as an integration of backscatter communications and the conventional cognitive radios, which is shown to achieve a higher throughput performance in [17]. The authors in [18] divided each time slot of the secondary user into three sub-phases for channel sensing, backscatter and active transmission, respectively. A Stackelberg game model is proposed to control the time resource allocation among different secondary users. The authors in [19] considered a backscatter-assisted wireless sensor network, in which each sensor node can optimize its time allocation between the passive and active transmissions. Given the time allocation between energy harvesting and the backscatter transmission, the authors in [20] analyzed the energy and information outage performance in a stochastic geometry framework. The optimal time allocation is also derived to maximize the network throughput. The authors in [21] proposed a hybrid cognitive radio network. The time allocation among ambient backscatter, bistatic backscatter, energy harvesting, and active communications can be optimized to maximize the throughput of the secondary communication system.

The user devices in different modes can also collaborate in data transmission. In particular, the passive radios can serve as wireless relays for the active RF communications. The author in [22] modeled the relay cooperation among multiple passive radios in a potential game. In [23], both the active and passive radios are allowed to serve as the relay for each other. The rate maximization problem is formulated to jointly optimize the time scheduling, power allocation, and energy beamforming strategies. The major difference with our work is that both the active and passive radios employ a half-duplex relay protocol. The relay node firstly receives the information from the source node, and then forwards it to the access point. The authors in [24] utilized the half-duplex relay to assist the information transmission between backscatter radios. The hybrid relay node in [25] can work in either active and passive relaying mode. In the active relaying mode, the first and second half of a time slot are allocated for the source-to- 
relay and relay-to-destination transmissions, respectively. The passive relaying mode is different during the second half of a time slot, by using backscatter communications to forward the data to the destination node.

\section{SYSTEM MODEL}

We consider a D2D network wirelessly powered by a multiantenna power beacon station (PBS) via RF signal beamforming. The set of all DUEs is denoted by $\mathcal{N}=\{1,2, \ldots, N\}$. As shown in Fig. 1, the PBS can be a dedicated power emitter for DUEs or the base station of an existing wireless system (e.g., the cellular network), which can be opportunistically used by the underlay D2D network as the energy source. The PBS can be viewed as the controller of the D2D network, scheduling the DUEs' data transmissions and radio mode switching. Each DUE is allocated a time slot $t_{n}$, for $n \in \mathcal{N}$, to transmit its information. The PBS and the base station can be connected and coordinated through the edge controller, which provides the capability of resource allocation, interference management, computation offloading, etc. The RF signals from the base station can also power the DUEs' underlay information transmissions. Depending on the spectrum activity of the base station, the PBS may schedule the DUEs' information transmissions in either passive or active mode. This can be viewed as an integration of cognitive and backscatter communications [17].

In this paper, assuming that the coordination between different networks can be well handled by the edge controller, we focus on the transmission performance of the wireless powered D2D network. Considering different channel conditions and power demands of individual DUEs, the multi-antenna PBS with $M$ antennas can vary its energy beamforming strategy to control the power transfer to different DUEs [2], with the aim of achieving the maximum sum throughput. Each DUE has only one antenna for either energy harvesting $(\mathrm{EH})$ or information transmission. The wireless powered D2D network can be envisioned as a wireless sensor network, in which the data gateway collects information from the sensor nodes and powers them by wireless power transfer. The complex channels from the PBS to the $n$-th DUE transmitter (DTx$n$ ) and the $n$-th DUE receiver $(\mathrm{DRx}-n)$ are denoted by $\mathbf{h}_{n}$ and $\mathbf{f}_{n}$, respectively. The channels from the DTx- $n$ to DTx- $m$ and DRx- $m$ are given by $z_{n, m}$ and $g_{n, m}$, respectively. Every channel is assumed to be frequency-flat block fading [26]. To exploit the radio diversity gain, we integrate two radio modes in the transceiver design. As shown in Fig. 1(b), the selection of radio modes and function modules is controlled by two RF switches $b(t)$ and $q(t)$, respectively. The binary variable $b(t)$ determines the radio mode, i.e., $b(t)=1$ indicates the active mode with matched load impedance $Z_{0}$ and $b(t)=0$ implies the passive mode with modulated load impedance $Z_{x}$.

\section{A. Energy Budget and Power Supply}

To supply energy for DUEs, we consider energy beamforming at the multi-antenna PBS and RF-based energy harvesting at the receiver, which converts the RF signals into DC voltage and then uses it to sustain wireless communications. Due to the

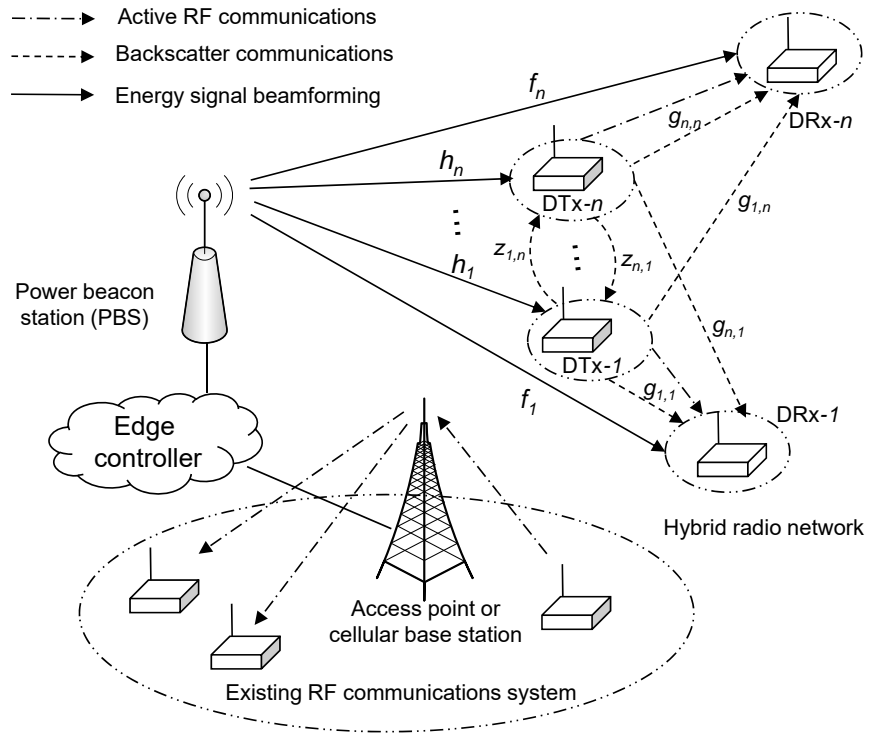

(a) System model.

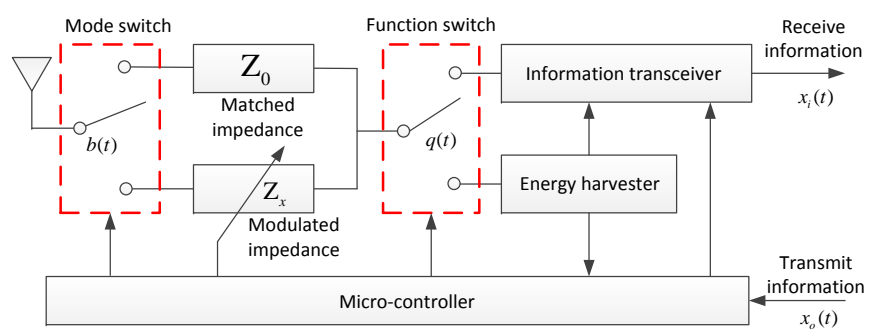

(b) Dual-mode radio.

Fig. 1: Hybrid network of active and passive radios.

broadcast nature of RF signals, the most appealing property is that it supports better mobility of the user devices and provides scalability for D2D networks [27]. The PBS can control the power transfer to different DUEs by adjusting its beamforming strategy. Meanwhile, each DUE can correspondingly adjust its EH parameters, e.g., the time-switching or power-splitting factor [2], to balance between its power demand and consumption. In this paper, we consider the timeswitching model that allows each DUE to harvest energy only when it is not scheduled for data transmission. Let $\mathbf{w}_{n}(t)$ denote the PBS' energy signal in the $n$-th time slot $t_{n}$ and $\mathbf{W}_{n} \triangleq \mathbb{E}\left[\mathbf{w}_{n}(t) \mathbf{w}_{n}(t)^{T}\right]$ be the transmit covariance. Thus, the PBS' beamforming strategy is subject to the feasible set defined as follows:

$$
\mathbb{P} \triangleq\left\{\begin{array}{l}
\sum_{n \in \mathcal{N}} t_{n} \operatorname{Tr}\left(\mathbf{W}_{n}\right) \leq E_{\max }, \text { and } \sum_{n \in \mathcal{N}} t_{n} \leq 1 \\
\operatorname{Tr}\left(\mathbf{W}_{n}\right) \leq P_{\max }, \quad \forall n \in \mathcal{N}
\end{array}\right.
$$

where $E_{\max }$ denotes the energy budget in one unit data frame and $P_{\max }$ is the PBS' peak transmit power. Here $\operatorname{Tr}(\cdot)$ denotes the trace of a matrix. We assume that the energy signal $\mathbf{w}_{n}(t)$ is generated by a fixed pattern known to the DUEs, and thus it will not introduce interference to the information reception by subtracting it from the received signals [27]. 


\section{B. Transmission Capability of Hybrid Radios}

With the dual-mode radio, each DTx- $n$ can choose either the passive or active mode depending on its available energy and the channel conditions. In the active mode, we assume perfect interference cancelation at the receiver. This allows us to subtract the known energy signal $\mathbf{w}_{n}(t)$ from the received signals. Hence, the throughput of DRx- $n$ is given by

$$
r_{n}^{A}=t_{n} \log \left(1+\left|g_{n, n}\right|^{2} p_{n}\right) .
$$

Here we assume normalized noise power to simplify the formulation. It is obvious that the throughput $r_{n}^{A}$ is nonconvex in $\left(t_{n}, p_{n}\right)$, where the transmit power $p_{n}$ depends on the beamforming strategies in all other time slots. This implies close couplings between different DUEs.

In the passive mode, the throughput is achieved by reflecting and modulating the RF signals emitted by the PBS. Given the PBS' energy signal $\mathbf{w}_{n}(t)$, the signal received at DTx- $n$ is given as $d_{n}(t)=\mathbf{h}_{n}^{H} \mathbf{w}_{n}(t)$. Meanwhile, DTx- $n$ modulates its information $s(t)$ on the carrier signal $d_{n}(t)$ by controlling the load-dependent reflection coefficient $\Gamma_{n, n}$. Hence, the backscattered signal is a joint effect of the load-dependent scattering and the load-independent structural scattering [7]. We can denote it as $s(t)=A_{s}-\Gamma_{n, n}$ where constant $A_{s}$ represents the effect of structural scattering. The backscattered signal is further attenuated by the channel $g_{n, n}$ from DTx- $n$ to DRx $-n$. Hence, the signal received by DRx- $n$ is given by:

$$
y_{n}(t)=g_{n, n} \mathbf{h}_{n}^{H} \mathbf{w}_{n}(t)\left(A_{s}-\Gamma_{n, n}\right)+\mathbf{f}_{n}^{H} \mathbf{w}_{n}(t)+v_{n},
$$

where $v_{n} \backsim \mathcal{C N}(0,1)$ denotes the normalized baseband noise in information reception. The first term contains the modulated information symbol $s(t)=A_{s}-\Gamma_{n, n}$ to the receiver, which is obviously not Rayleigh distributed. This significantly complicates the evaluation of the channel capacity.

Though an exact and closed-form expression of channel capacity is not available for backscatter communications, the authors in [5] have showed that the capacity of backscatter communications can be upper bounded by a log function, which is increasing and concave with respect to the averaged SNR performance of backscatter communications. Such an upper bound can be achieved when the backscatter receiver is able to reliably estimate the legacy symbols and counteract the interference generated by the legacy transmission. As such, we characterize the throughput in the passive mode as follows:

$$
r_{n}^{P}=t_{n} \log \left(1+\tilde{m}_{s}\left|g_{n, n}\right|^{2} \mathbf{h}_{n}^{H} \mathbf{W}_{n} \mathbf{h}_{n}\right),
$$

where we denote $\tilde{m}_{s}$ as the average power of the backscattered signal $s(t)$. It can be set to unit one without loss of generality. A similar log-function has also been adopted in [28] and [29].

\section{Backscatter-aided Relay Communications}

When DTx-r has sufficient energy supply, it can turn to the passive mode and assist data transmissions for some other user, e.g., DTx- $n$, especially for those with insufficient energy supply and low data rate. The users' cooperation may significantly increase the throughput of DTx-n, while compromising little to the throughput of DTx- $r$. As illustration in Fig. 2, considering the data transmission of DTx- $n$, the other users can either harvest energy from the PBS or turn to passive mode to assist DTx- $n$. DTx- $r$ may have a good channel condition or high energy harvesting rate. It prefers to relay data transmissions for DTx- $n$ instead of harvesting energy. While DTx- $k$ demands more energy, it decides not to relay for DTx$n$, but to exploit the energy harvesting opportunities.

Let $\mathcal{R}_{n}$ denote the set of backscatter relays for DTx- $n$ in the first sub-slot $t_{n, 1}$. Each DTx- $r$, for $r \in \mathcal{R}_{n}$, will reflect the incident signals with the reflection coefficient $\Gamma_{r, n}$. The received signals at DRx $n$ are a mixture of the signals directly from the DTx- $n$ and the relays' reflections. In particular, the $\mathrm{RF}$ signal $d_{n, r}$ at DTx- $r$ is given by

$$
d_{n, r}=z_{n, r} \sqrt{p_{n, 1}} s_{n}(t)+\mathbf{h}_{r}^{H} \mathbf{w}_{n, 1}(t)+v_{n, r},
$$

where $s_{n}(t)$ is the signal transmitted from DTx- $n$ to DRx- $n$ and $v_{n, r}$ denotes the complex Gaussian noise at the antenna. The second term denotes the direct beamforming from the PBS to DTx- $r$. After DTx-r's backscattering, the signal received at DRX- $n$ can be simply denoted as

$$
y_{r, n}=\Gamma_{r, n} g_{r, n} z_{n, r} \sqrt{p_{n, 1}} s_{n}(t)+\mathbf{I}_{r, n}^{H} \mathbf{w}_{n, 1}(t)+\sigma_{n, 1},
$$

where $\sigma_{n, 1} \backsim \mathcal{C N}(0,1)$ is the normalized noise and $\mathbf{I}_{r, n} \triangleq$ $\Gamma_{r, n} g_{r, n} \mathbf{h}_{r}+\mathbf{f}_{n}$ is viewed as the equivalent interference channel related to the reflection coefficient $\Gamma_{r, n}$ of the relay DTx- $r$. Thus, the second term, $\mathbf{I}_{r, n}^{H} \mathbf{w}_{n, 1}(t)$, denotes the interference due to the PBS' energy beamforming. We assume that the users can exchange information at the beginning of each data transmission. We assume that the receiver DTx- $r$ has dedicated circuit design and signal processing capability than conventional passive devices, e.g., RFID tags. It can be configured with the capability to subtract interference signals directly from the PBS. A similar idea has also been adopted in some existence works, e.g., [30] and [28]. Once DTx- $r$ sets its reflection coefficient $\Gamma_{r, n}$ and informs it to DRx- $n$, the receiver DRx- $n$ can estimate the interference term $\mathbf{I}_{r, n}^{H} \mathbf{w}_{n, 1}(t)$ and subtract it from the received signals. When multiple relays work together, the signal received at DRx- $n$ after interference cancelation is given by:

$$
\tilde{y}_{n}=\sqrt{p_{n, 1}}\left(\sum_{r \in \mathcal{R}_{n}} \Gamma_{r, n} g_{r, n} z_{n, r}+g_{n, n}\right) s_{n}(t)+\sigma_{n, 1} .
$$

Let $\tilde{g}_{n, n}\left(\mathcal{R}_{n}\right) \triangleq g_{n, n}+\sum_{r \in \mathcal{R}_{n}} \Gamma_{r, n} g_{r, n} z_{n, r}$ denote the equivalent relay channel from DTx- $n$ to DRx- $n$, which is enhanced by the set of passive relays in $\mathcal{R}_{n}$. Hence, the throughput of DTx- $n$ in active mode with the assistance from the passive relays can be characterized by

$$
\tilde{r}_{n}^{A}=t_{n, 1} \log \left(1+e_{n, 1}\left|\tilde{g}_{n, n}\left(\mathcal{R}_{n}\right)\right|^{2} / t_{n, 1}\right) .
$$

By properly choosing the relays' reflection coefficients according to their channel conditions, we can build constructive multi-path effect to enhance the signal transmission to DRx$n$. The adaptation of each relay's reflection coefficient can be achieved by constructing a finite set of load impedances connected by a multi-throw RF switch [7]. The optimal reflection coefficient can be chosen by controlling the RF switch to pass signals through the corresponding load impedance. 


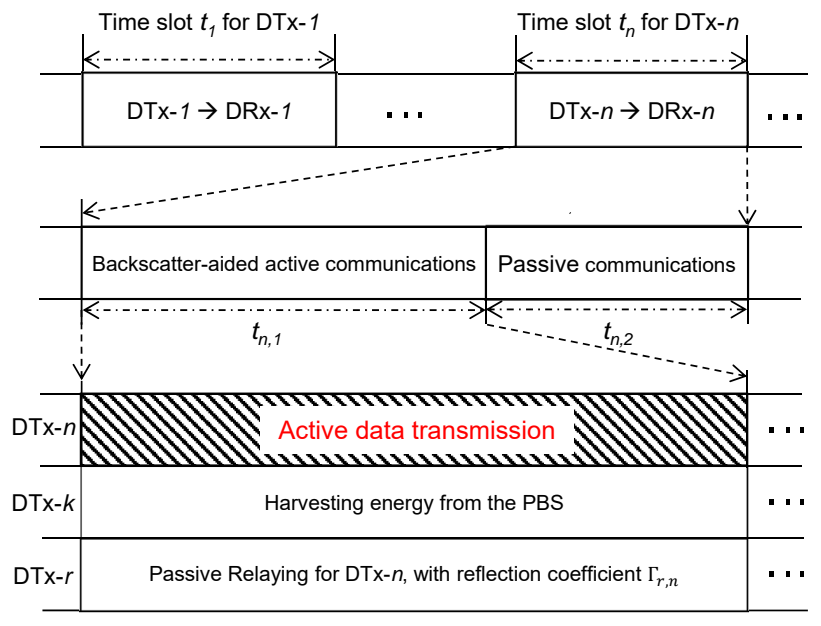

Fig. 2: Time-slotted hybrid channel access.

In the sequel, we first propose the sum throughput maximization problem to exploit the radio diversity gain, and then present the reformulation to further improve the performance gain by leveraging the users' cooperation among hybrid radios.

\section{Throughrut MaXimization Via Mode SElection}

Note that each DUE can transmit in two radio modes with different transmission capabilities, as shown in (2) and (3), respectively. To maximize the sum throughput, we jointly optimize the DUEs' radio modes, the PBS' beamforming and time allocation strategies. Let $b_{n} \in\{0,1\}$ indicate whether DTx- $n$ is in the active mode (i.e., $b_{n}=1$ indicates the active mode) and denote $p_{n}$ as the transmit power of DTx- $n$ in active mode, which relies on its energy harvesting in other time slots $t_{m}$, for $m \in \mathcal{N}_{n} \triangleq \mathcal{N} \backslash\{n\}$. Hence, the power budget constraint of DTx- $n$, for $n \in \mathcal{N}$, is given as follows:

$$
b_{n} p_{n} t_{n} \leq \sum_{m \in \mathcal{N}_{n}} \eta\left(t_{m} \mathbf{h}_{n}^{H} \mathbf{W}_{m} \mathbf{h}_{n}+b_{m}\left|z_{m, n}\right|^{2} p_{m} t_{m}\right),
$$

where $\eta$ denotes the constant energy conversion efficiency and $z_{m, n}$ denotes the channel from the $m$-th transmitter to the $n$-th transmitter. The first term in RHS of (6) represents the energy directly harvested from the power beacon station, while the second term (i.e., $b_{m}\left|z_{m, n}\right|^{2} p_{m} t_{m}$ ) is the energy harvested from the active radios nearby DTx- $n$.

Note that we omit the circuit power consumption in (6). However, it can be easily extended by adding a constant term to describe the circuit power consumption, which will not change the following solution method. We also assume that the user's energy will be depleted after data transmission. Hence, each user accumulates all energy harvested in the past $N-1$ time slots before the data transmission in the current time slot. For each DTx- $n$, we construct $\mathbf{a}_{n}=\left[a_{n 1}, a_{n 2}, \ldots, a_{n N}\right]^{T}$ such that $a_{n n}=b_{n}$ and $a_{n m}=-\eta b_{m}\left|g_{m, n}\right|^{2}$ for $m \in \mathcal{N}_{n}$. Then we can simplify (6) as follows:

$$
\sum_{m \in \mathcal{N}} a_{n m} p_{m} t_{m} \leq \eta \sum_{m \in \mathcal{N}_{n}} t_{m} \mathbf{h}_{n}^{H} \mathbf{W}_{m} \mathbf{h}_{n}, \forall n \in \mathcal{N} .
$$

It is obvious that (7) defines a linear inequality with a fixed radio mode $\mathbf{b}=\left[b_{1}, b_{2}, \ldots, b_{N}\right]^{T}$.
One point worth mentioning is that we actually employ a linear EH model in (6) and (7), which is characterized by the constant $\eta$. That is, the total harvested energy is linearly dependent on the strength of incident RF signals at the receiver, i.e., $\sum_{m \in \mathcal{N}_{n}} t_{m} \mathbf{h}_{n}^{H} \mathbf{W}_{m} \mathbf{h}_{n}$. A more practical nonlinear EH model has been presented in [31], in which $\eta$ is measured to be a function of the RF signal strength. In this case, the power budget constraint (7) becomes more complicated and generally non-convex. As such, we can apply successive convex approximation (SCA) algorithm that replaces (7) by a linear form in each iteration. A similar approach has been applied in [32]. By introducing auxiliary variables $\mathbf{E}_{n} \triangleq t_{n} \mathbf{W}_{n}$ and $e_{n} \triangleq p_{n} t_{n}$, for $n \in \mathcal{N}$, we can formulate the sum throughput maximization as follows ${ }^{1}$ :

$$
\begin{aligned}
\max _{\mathbf{b}, \mathbf{e},\left(\mathbf{t}, \mathbf{E}_{n}\right) \in \mathbb{P}} & \sum_{n \in \mathcal{N}} t_{n} \log \left(1+\left|g_{n, n}\right|^{2} \tilde{e}_{n} / t_{n}\right) \\
\text { s.t. } & \tilde{e}_{n} \leq b_{n} e_{n}+\left(1-b_{n}\right) \mathbf{h}_{n}^{H} \mathbf{E}_{n} \mathbf{h}_{n}, \forall n \in \mathcal{N} \\
& \mathbf{a}_{n}^{T} \mathbf{e} \leq \eta \mathbf{h}_{n}^{H} \mathbf{E}_{-n} \mathbf{h}_{n}, \forall n \in \mathcal{N},
\end{aligned}
$$

where we denote $\mathbf{e}=\left[e_{1}, e_{2}, \ldots, e_{N}\right]^{T}$ and $\mathbf{E}_{-n}=$ $\sum_{m \in \mathcal{N}_{n}} \mathbf{E}_{m}$ for convenience. The variable $\tilde{e}_{n}$ is viewed as the equivalent transmit power of DTx- $n$, which depends on the radio mode $b_{n}$. When $b_{n}=1\left(\right.$ or $\left.b_{n}=0\right)$ and DTx- $n$ operates in the active (or passive) mode, we have $\tilde{e}_{n}=t_{n} p_{n}$ (or $\tilde{e}_{n}=t_{n} \mathbf{h}_{n}^{H} \mathbf{W}_{n} \mathbf{h}_{n}$ ). Hence, the constraint (8b) actually integrates the transmission capabilities in two modes.

It is obvious that problem (8) is a mixed integer non-linear program (MINLP). For fixed radio mode $\mathbf{b}$, each constraint in (8b)-(8c) becomes linear in $\left(\mathbf{t}, \mathbf{E}_{n}, \mathbf{e}\right)$. Thus, we can easily verify that the problem (8) is convex by checking the Hessian matrix of (8a). To optimize the binary $\mathbf{b}$, we can consider a branch-and-bound (BB) algorithm [33], which requires the evaluations of lower and upper bounds of the problem (8). The convergence can be achieved by successively improving the lower bound and trimming undesired branches. A lower bound of (8) can be easily obtained by fixing the DUEs' radio modes and solving a convex problem by an existing optimization toolbox [34]. However, the evaluation of upper bound becomes more challenging. Typically, the upper bound can be achieved by simply relaxing the binary $\mathbf{b}$ into continuous $\hat{\mathbf{b}} \in[\mathbf{0}, \mathbf{1}]$. Unfortunately, this relaxation leads to another non-convex problem due to the quadratic couplings in (8b) and (8c). As such, we consider an ideal case in which the radio mode switching can happen any time during the data transmission. In particular, we propose the hybrid access model that splits each time slot into two sub-slots for active and passive radios, respectively. This mechanism makes it efficient to evaluate an upper bound of (8).

\section{A. Upper Bound via Hybrid Access Model}

In the hybrid access model, each time slot $t_{n}$ is further divided into two sub-slots. One part $t_{n, 1}$ is used for data transmissions in the active mode and the other sub-slot $t_{n, 2}$ is

\footnotetext{
${ }^{1}$ We only enumerate a few variables of our interest, i.e., $\left(\mathbf{t}, \mathbf{E}_{n}, \mathbf{e}\right)$, as the decision variables of problem (8). For simplicity, the auxiliary or intermediate variables are omitted in the list of decision variables. The same convention also applies to the following problems, e.g., (10), (11), and (15).
} 
for the passive backscatter communications. Correspondingly, we define $\mathbf{w}_{n, i}(t)$ as the PBS' energy signal and $\mathbf{W}_{n, i}$ as the PBS' transmit covariance in the sub-slot $t_{n, i}$, for $i \in\{1,2\}$ and $n \in \mathcal{N}$. Hence, the feasible set (1) is rewritten as follows:

$$
\left\{\begin{array}{l}
\sum_{n \in \mathcal{N}}\left(t_{n, 1} \operatorname{Tr}\left(\mathbf{W}_{n, 1}\right)+t_{n, 2} \operatorname{Tr}\left(\mathbf{W}_{n, 2}\right)\right) \leq E_{\max } \\
\operatorname{Tr}\left(\mathbf{W}_{n, i}\right) \leq P_{\max }, \quad \forall n \in \mathcal{N} \text { and } i \in\{1,2\}
\end{array}\right.
$$

Our design objective is to maximize the sum throughput by optimizing the PBS' energy beamforming strategies $\mathbf{W} \triangleq\left\{\mathbf{W}_{n, i}\right\}_{n \in \mathcal{N}, i \in\{1,2\}}$, the DUEs' transmit power $\mathbf{p}=$ $\left[p_{n, 1}, p_{n, 1}, \ldots, p_{N, 1}\right]^{T}$ in the active mode and the transmission scheduling strategy $\mathbf{t} \triangleq\left(\mathbf{t}_{1}, \mathbf{t}_{2}\right)$ in two radio modes.

Similar to (8), by setting $\mathbf{E}_{n, i} \triangleq t_{n, i} \mathbf{W}_{n, i}$ and $e_{n, i} \triangleq$ $p_{n, i} t_{n, i}$, for $n \in \mathcal{N}$ and $i \in\{1,2\}$, we can reformulate the sum throughput maximization problem as follows:

$$
\begin{aligned}
\max _{\mathbf{t}, \mathbf{E}_{n, i}, e_{n, i}} & \sum_{n \in \mathcal{N}, i \in\{1,2\}} t_{n, i} \log \left(1+\left|g_{n, n}\right|^{2} e_{n, i} / t_{n, i}\right) \\
\text { s.t. } & e_{n, 1} \leq \sum_{i \in\{1,2\}} \eta \mathbf{h}_{n}^{H} \mathbf{E}_{-n, i} \mathbf{h}_{n}, \\
& e_{n, 2} \leq \mathbf{h}_{n}^{H} \mathbf{E}_{n, 2} \mathbf{h}_{n}, \\
& \sum_{i \in\{1,2\}} \operatorname{Tr}\left(\mathbf{E}_{n, i}+\mathbf{E}_{-n, i}\right) \leq E_{\max }, \\
& \operatorname{Tr}\left(\mathbf{E}_{n, i} \leq t_{n, i} P_{\max }, \text { and } \mathbf{t}_{1}+\mathbf{t}_{2} \leq \mathbf{1},\right. \\
& i \in\{1,2\} \text { and } n \in \mathcal{N},
\end{aligned}
$$

where we define $\mathbf{E}_{-n, i} \triangleq \sum_{m \in \mathcal{N}_{n}} \mathbf{E}_{m, i}$. Note that the transmit power of different DUE is in a similar range, and much smaller than that of the PBS. Each DUE's energy harvesting mainly comes from the PBS and hence we simplify the power budget constraint as in (10b). Besides, we impose a per-user time constraint as in (10e) instead of the sum time constraint $\sum_{n \in \mathcal{N}} t_{n} \leq 1$ as in (1), which shares unit data frame among all DUEs. The time constraint in (10e) allocates each DTx- $n$ a unit time for data transmission, and allows flexible transmission scheduling between two radio modes. It can also be modified to account for the total time requirement.

By checking the first- and second-derivatives, the objective in (10a) is increasing and concave in $\left(t_{n, i}, e_{n, i}\right)$. We further notice that all constraints are linear inequalities. Hence, problem (10) can be efficiently solved by the interiorpoint algorithms [35]. Once we obtain the optimal solution $\left(\mathbf{t}^{\star}, \mathbf{E}_{n, i}^{\star}, e_{n, i}^{\star},\right)$, the optimal beamforming strategy in each sub-slot is given by $\mathbf{W}_{n, i}^{\star}=\mathbf{E}_{n, i}^{\star} / t_{n, i}^{\star}$ if $t_{n, i}^{\star}>0$ and $\mathbf{W}_{n, i}^{\star}=\mathbf{0}$ if $t_{n, i}^{\star}=0$. The transmit power $p_{n, 1}$ in the active mode can be extracted from $e_{n, 1}^{\star}$ similarly. Most importantly, problem (10) provides an efficient method to evaluate the upper bound of problem (8). In fact, problem (8) can be viewed as a special case of problem (10) when each DTx- $n$ only transmits with one radio mode during the whole time slot, i.e., either $t_{n, 1}=1$ or $t_{n, 1}=0$. If the optimal time allocation to problem (10) happens to be binary, it provides a tight upper bound on problem (8).

\section{B. Optimal Radio Mode Selection}

The upper bound of (8) can be evaluated by relaxing each binary variable $b_{n}$ into its continuous counterpart $\hat{b}_{n} \in[0,1]$.
Though the relaxed problem is still non-convex and difficult to solve directly, the hybrid access model allows us to derive a convex reformulation in (10). As such, we can simply determine the optimal solution $\hat{\mathbf{b}}^{*} \in[\mathbf{0}, \mathbf{1}]$ by the optimal transmission scheduling strategy $\mathbf{t}^{*}$ in $(10)$, i.e., $\hat{\mathbf{b}}^{*}=\mathbf{t}^{*}$. If $\hat{\mathbf{b}}^{*}$ happens to be binary, the optimal radio mode is given by $\mathbf{b}^{*}=\hat{\mathbf{b}}^{*}$. Otherwise, for each non-binary $\hat{b}_{n}^{*}$, we will divide the feasible region of $\left(\hat{b}_{n}, \hat{\mathbf{b}}_{-n}\right)$ into two sub-divisions, i.e., $D_{n}^{0}=\left\{\left(0, \hat{\mathbf{b}}_{-n}\right)\right\}$ and $D_{n}^{1}=\left\{\left(1, \hat{\mathbf{b}}_{-n}\right)\right\}$, where $\hat{\mathbf{b}}_{-n}$ denotes the relaxed mode selection of all DUEs rather than DTX- $n$ and it can take any value from the set $[\mathbf{0 , 1}]$. Then we continue to evaluate the lower and upper bounds on $D_{n}^{0}$ and $D_{n}^{1}$, respectively. A sub-division can be discarded if it produces an upper bound less than the current optimum of (8). The detailed algorithm follows a similar procedure as that proposed in our previous work [36]. The new enhancement in this work lies in that we provide a tight upper bound of problem (8) by solving a convex throughput maximization problem efficiently, instead of the approximate upper bound by a heuristic method.

Till this point, we can find optimal solutions for both radio mode selection problem (8) and the mode switching problem (10). The optimal mode switching provides the basis for iterative updating the DUEs' radio mode selection in the branch-and-bound algorithm. Obviously, the radio selection problem has much higher computational complexity, while the radio switching problem requires more coordination and overhead for practical implementation. In particular, to realize online mode switching, each DTx- $n$ requires precise control on its radio mode according to the optimized transmission scheduling strategy. This implies strict synchronization between DTx- $n$ and DRx- $n$, e.g., DRx- $n$ may need to adjust its decoding scheme corresponding to different radio mode of DTX- $n$. The synchronization with the PBS is also required as the beamforming strategies may change over two sub-slots, along with the radio mode switching.

\section{ENHANCED TRANSMISSION VIA BACKSCATTER RELAY COMMUNICATIONS}

After solving problem (10), each DTx- $n$ will transmit in the active and passive modes sequentially for the optimized subslots, and harvest RF power in other time slots to accumulate energy for active communications in the subsequent time slots. In the sequel, we first provide a problem reformulation to account for the users' cooperation, and then devise both approximate and heuristic algorithms to optimize the hybrid radios' relaying strategies.

Let $\mathcal{R}_{n}$ denote the set of backscatter relays for DTx$n$ in the first sub-slot $t_{n, 1}$. Each DTx- $r$, for $r \in \mathcal{R}_{n}$, will reflect the incident signals with the reflection coefficient $\Gamma_{r, n}$. When multiple relays work together, the throughput of DTX- $n$ in active mode with the assistance from the passive relays can be characterized by (5). Let $o_{n} \triangleq\left|\tilde{g}_{n, n}\left(\mathcal{R}_{n}\right)\right|^{2}$ denote the enhanced channel gain of DTx- $n$. Then, we can rewrite the throughput $\tilde{r}_{n}^{A}$ in the active mode as $\tilde{r}_{n}^{A}=$ $t_{n, 1} \log \left(1+e_{n, 1} o_{n} / t_{n, 1}\right)$, and correspondingly reformulate 
the sum throughput maximization (10) as follows:

$$
\begin{aligned}
& \max _{\mathbf{t}, \mathbf{E}_{n, i}, \Gamma_{r, n}} \sum_{n \in \mathcal{N}}\left(\tilde{r}_{n}^{A}+r_{n}^{P}\right) \\
& \text { s.t. } o_{n} \leq\left|g_{n, n}+\sum_{r \in \mathcal{N}_{n}} \Gamma_{r, n} g_{r, n} z_{n, r}\right|^{2} \\
& e_{n, 1} \leq \sum_{r \in \mathcal{N}_{n}} \eta\left(\left(1-\left|\Gamma_{n, r}\right|^{2}\right) \alpha_{n, r}+\beta_{n, r}\right) \\
&(10 \mathrm{c})-(10 \mathrm{e}), \forall r \in \mathcal{N}_{n} \text { and } n \in \mathcal{N}
\end{aligned}
$$

where we simply denote $\alpha_{n, r} \triangleq \mathbf{h}_{n}^{H} \mathbf{E}_{r, 1} \mathbf{h}_{n}$ and $\beta_{n, r} \triangleq$ $\mathbf{h}_{n}^{H} \mathbf{E}_{r, 2} \mathbf{h}_{n}$ as the RF power at DTx- $n$ in the sub-slot $t_{r, 1}$ and $t_{r, 2}$, respectively. In problem (11), we also optimize the passive relaying strategy of the hybrid radio network, which dictates each DUE's reflection coefficient $\Gamma_{r, n}$, for $r \in \mathcal{N}_{n}$ and $n \in \mathcal{N}$. However, the optimization of the relay set $\mathcal{R}_{n}$ is challenging due to the combinatorial nature. Instead, we view $\Gamma_{r, n}$ as the continuous optimization variable. A higher value of $\left|\Gamma_{r, n}\right|$ implies that DTX- $r$ takes more effort to relay information for DTx- $n$. The constraints in (11b) and (11c) allow free tuning of the reflection coefficient $\Gamma_{n, r}$ in its feasible region. When DTx- $n$ relays for DTx- $r$ with the reflection coefficient $\Gamma_{n, r}$, it can only harvest a part of energy from the incident RF signals, which is scaled by $1-\left|\Gamma_{n, r}\right|^{2}$ as shown in (11c). Note that we can tune the complex phase of $\Gamma_{r, n}$ to maximize the projection of the relay channel $g_{r, n} z_{n, r}$ on the backscatter channel $g_{n, n}$. As such, we only care about the optimization of its magnitude $\left|\Gamma_{r, n}\right|$ and thus we view $\Gamma_{r, n}$ as a real variable in (11).

\section{A. Successive Convex Approximation}

The main difficulty of problem (11) lies in that both (11b) and (11c) define non-convex sets. The throughput $\tilde{r}_{n}^{A}$ in the active mode is also non-convex due to the quadratic coupling between $e_{n, 1}$ and $o_{n}$. In addition, different choices of the relay's reflection coefficient lead to the throughput tradeoff between different DUE pairs. If DTX- $r$ chooses a high reflection level to enhance the channel $\tilde{g}_{n, n}\left(\mathcal{R}_{n}\right)$ of DTx- $n$, the throughput of DTx- $n$ can be improved correspondingly. However, this will sacrifice the energy harvesting opportunity $e_{r, 1}$ of DTx- $r$ and decrease its own throughput in the active mode. In the following, we propose the SCA-based iterative method to search for the sub-optimal solution to (11). In each iteration, we require a convex approximation of problem (11), whose solution will provide a new searching point in next iteration. We assume that the channel information is known at the beginning of each transmission period. The channel estimation can be performed at a dedicated time period before data transmission. In particular, the PBS can broadcast a pilot signal to all users, which can estimate the channels $\mathbf{h}_{n}$ locally and report the estimates to the PBS in a time-slotted manner. During the reporting periods, other overhearing radios can estimate the channels $z_{n, m}$ and $g_{n}$ simultaneously. A similar idea has been proposed in [37].

To approximate (11c), we introduce a set of auxiliary variables $x_{n, r}$ and $y_{n, r}$ such that $y_{n, r} \leq 1-\left|\Gamma_{n, r}\right|^{2}$ and $x_{n, r}^{2} \leq y_{n, r} \alpha_{n, r}$. Then, we can rewrite (11c) as $e_{n, 1} \leq$

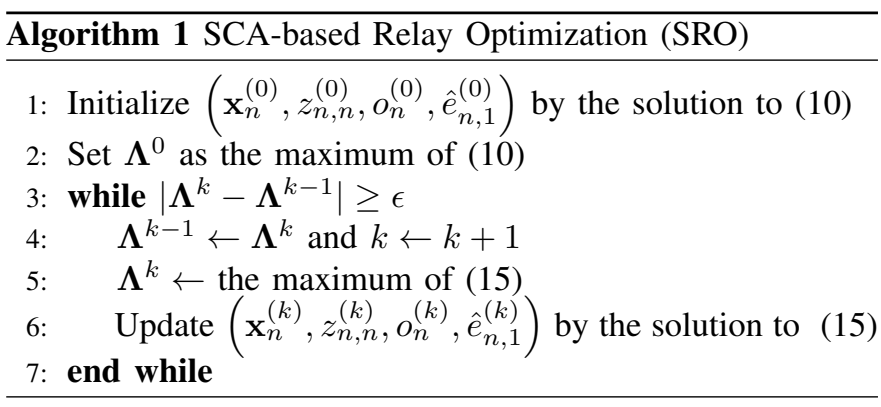

$\sum_{r \in \mathcal{N}_{n}} \eta\left(x_{n, r}^{2}+\beta_{n, r}\right)$, which admits a first-order linear approximation as follows:

$$
2\left(\mathbf{x}_{n}^{(k)}\right)^{T} \mathbf{x}_{n}-\left|\mathbf{x}_{n}^{(k)}\right|^{2} \geq \eta^{-1} e_{n, 1}-\beta_{n},
$$

where $\mathbf{x}_{n} \triangleq\left[x_{n, r}\right]_{r \in \mathcal{N}_{n}}$ and $\beta_{n}=\sum_{r \in \mathcal{N}_{n}} \beta_{n, r}$ denotes the total energy harvested by DTx $n$ in the second subslots. The constant $\mathbf{x}_{n}^{(k)}$ denotes the value of $\mathbf{x}_{n}$ at the $k$ th iteration. Similar, define auxiliary variable $z_{n, n}$ such that $z_{n, n} \leq \sum_{r \in \mathcal{N}_{n}} \Gamma_{r, n} g_{r, n} z_{n, r}$, then the constraint in (11b) can be linearly approximated as follows:

$$
o_{n} \leq o_{n}^{(k)}+2\left|g_{n, n}+z_{n, n}^{(k)}\right|\left(z_{n, n}-z_{n, n}^{(k)}\right),
$$

where $o_{n}^{(k)} \triangleq\left|g_{n, n}+z_{n, n}^{(k)}\right|^{2}$. To approximate the nonconvex $\tilde{r}_{n}^{A}$ in (11a), we introduce $\hat{o}_{n}$ such that $\hat{o}_{n} \leq e_{n, 1} o_{n}$ and then rewrite it as $\tilde{r}_{n}^{A}=t_{n, 1} \log \left(1+\hat{o}_{n} / t_{n, 1}\right)$, which is easily shown to be concave in $\left(t_{n, 1}, \hat{o}_{n}\right)$. Moreover, by rewriting $e_{n, 1} o_{n}$ as the sum of a concave and a convex function, i.e., $e_{n, 1} o_{n}=\left[\left(o_{n}+e_{n, 1}\right)^{2}-\left(o_{n}-e_{n, 1}\right)^{2}\right] / 4$, we can approximate the non-convex constraint $\hat{o}_{n} \leq e_{n, 1} o_{n}$ as follows:

$$
2 \hat{e}_{n, 1}^{(k)} \hat{e}_{n, 1}-\left(\hat{e}_{n, 1}^{(k)}\right)^{2} \geq 4 \hat{o}_{n}+\left(o_{n}-e_{n, 1}\right)^{2},
$$

where we define $\hat{e}_{n, 1} \triangleq o_{n}+e_{n, 1}$ and $\hat{e}_{n, 1}^{(k)} \triangleq o_{n}^{(k)}+e_{n, 1}^{(k)}$. Till this point, at the $k$-th iteration of the SCA-based method, we can solve (11) by the following convex problem:

$$
\begin{aligned}
\max _{\mathbf{t}, \mathbf{E}_{n, i}, \Gamma_{r, n}} & \sum_{n \in \mathcal{N}}\left(\tilde{r}_{n}^{A}+r_{n}^{B}\right) \\
\text { s.t. } & y_{n, r} \leq 1-\left|\Gamma_{n, r}\right|^{2}, \forall r \in \mathcal{N}_{n} \text { and } n \in \mathcal{N} \\
& {\left[\begin{array}{cc}
y_{n, r} & x_{n, r} \\
x_{n, r} & a_{n, r}
\end{array}\right] \succeq 0, \forall r \in \mathcal{N}_{n} \text { and } n \in \mathcal{N} } \\
& (10 \mathrm{c})-(10 \mathrm{e}), \text { and }(12)-(14) .
\end{aligned}
$$

The resulting problem is a semi-definite program (SDP), which can be solved efficiently by the interior-point algorithms [35]. A special case of problem (15) is to assume that all DUEs set the same reflection coefficient, i.e., $\Gamma=\Gamma_{r, n}$ for $r \in \mathcal{N}_{n}$ and $n \in \mathcal{N}$. In this case, we can simplify (15) by similarly constructing $y \leq 1-\Gamma^{2}$ and $x_{n}^{2} \leq y \alpha_{n} \triangleq y \sum_{r \in \mathcal{N}_{n}} \alpha_{n, r}$. The benefit of this special case mainly lies in the reduced number of decision variables as well as the high computational complexity for solving SDPs [38].

The initial value $\left(\mathbf{x}_{n}^{(k)}, z_{n, n}^{(k)}, o_{n}^{(k)}, \hat{e}_{n, 1}^{(k)}\right)$ for $k=0$ can be simply found by solving the convex problem (10), in which the passive relaying strategy is disenabled, i.e., $\left|\Gamma_{r, n}\right|=0$ 
for $r \in \mathcal{N}_{n}$ and $n \in \mathcal{N}$. Hence, we can set $x_{n, r}^{(0)}=$ $\left(\alpha_{n, r}\right)^{1 / 2}=\left(\mathbf{h}_{n}^{H} \mathbf{E}_{r, 1} \mathbf{h}_{n}\right)^{1 / 2}$ where $\mathbf{E}_{r, 1}$ is the solution to (10). Similar, we have $o_{n}^{(0)}=\left|g_{n, n}+\sum_{r \in \mathcal{N}_{n}} g_{r, n} z_{n, r}\right|^{2}$ and $\hat{e}_{n, 1}^{(0)}=o_{n}^{(0)}+\sum_{r \in \mathcal{N}_{n}} \eta\left(\alpha_{n, r}+\beta_{n, r}\right)$. Given the starting point, the SCA-based relay optimization (SRO) scheme, as shown in Algorithm 1, will find convex approximations (12)-(14) for the objective and non-convex constraints. The solution to (15) will then help update the search point in next iteration. This process continues until the convergence to a sub-optimum. Our simulation results verify that the SRO algorithm converges within a few iterations. In each iteration, the SRO algorithm requires to solve an SDP (15), which can be efficiently solve by the interior-point algorithm. By the theoretical analysis in [38], the computational complexity of an SDP problem can be bounded by a polynomial of the number of linear matrix inequalities and the size of matrix variables.

\section{B. Heuristic Passive Relaying Strategies}

The SRO scheme in Algorithm 1 implements an iterative procedure with guaranteed convergence to a sub-optimal solution. However, it still has a high computational complexity as we require to solve an SDP (15) in each iteration. Note that problem (15) has a large set of matrix inequalities in (15c) as well as intermediate decision variables. All these make the SRO scheme very inefficient in practice. In the following, we devise a set of heuristic algorithms to find a practical passive relaying strategy for each DUE in the hybrid radio network.

1) Myopic Relay Cooperation $(\mathrm{MRC})$ : The most straightforward relay scheme is to enforce full cooperation among all DUEs. That is, all DUEs set their maximum reflection coefficients $\Gamma_{r, n}^{\max }$ to relay information of the active radios. The energy harvesting will only take place in the second subslots. Given the DUEs' energy harvesting capabilities, this relay strategy in fact myopically maximizes the throughput of active radios in the first sub-slots. Thus, we can simplify the throughput maximization problem (11) as follows:

$$
\begin{aligned}
\max _{\mathbf{t}, \mathbf{E}_{n, i}} & \sum_{n \in \mathcal{N}}\left(\tilde{r}_{n}^{A}+r_{n}^{P}\right) \\
\text { s.t. } & o_{n} \leq\left|g_{n, n}+\sum_{r \in \mathcal{R}_{n}} \Gamma_{r, n}^{\max } g_{r, n} z_{n, r}\right|^{2} \\
& e_{n, 1} \leq \sum_{r \in \mathcal{N}_{n}} \eta\left(\left(1-\left|\Gamma_{r, n}^{\max }\right|^{2}\right) \alpha_{n, r}+\beta_{n, r}\right), \\
& (10 \mathrm{c})-(10 \mathrm{e}), \forall r \in \mathcal{N}_{n} \text { and } n \in \mathcal{N} .
\end{aligned}
$$

It is obvious that (16) has a similar form as that in (10). Hence, the optimal joint strategy $\left(\mathbf{t}, \mathbf{E}_{n, i}\right)$ can be optimized efficiently. The throughput performance achieved by the MRC scheme can serve as a baseline (i.e., lower bound) for performance comparison with more advanced schemes.

2) Equal Residual Energy (ERE): The main difficulty of problem (11) lies in the quadratic coupling between the channel gain $o_{n}$ and the energy $e_{n, 1}$ in the objective (11a). In this part, we design the ERE scheme that stems from the intuition that any DTx- $r$ will take more effort to relay information for DTX- $n$ if it can harvest more energy in

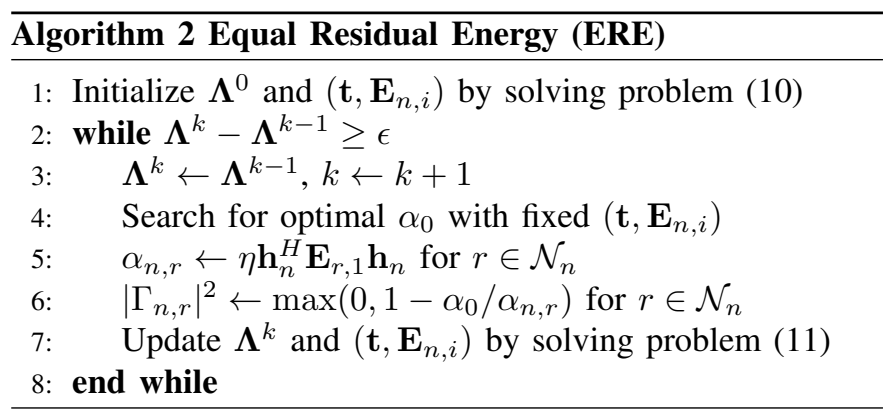

the time slot $t_{n}$ allocated for DTx- $n$. That is, the passive relaying scheme becomes a fair trade, i.e., if some DTx$r$ receives stronger RF power in time slot $t_{n}$, it can set a correspondingly larger reflection coefficient $\Gamma_{r, n}$ as a return to assist the data transmission of DTx- $n$. The residual energy, i.e., $\left(1-\left|\Gamma_{n, r}\right|^{2}\right) \alpha_{n, r}$ as seen from (11c), is the difference between the incident RF power and the reflected power, which can be viewed as the net profit of DTx- $r$ when it relays for DTx- $n$.

The ERE scheme ensures that each passive relay DTx- $r$ intercepts the same residual energy $\alpha_{0}=\left(1-\left|\Gamma_{n, r}\right|^{2}\right) \alpha_{n, r}$, for $r \in \mathcal{N}_{n}$. However, the optimal value $\alpha_{0}$ needs to be carefully chosen to maximize the overall throughput performance. With a fixed $\alpha_{0}$, we can simply determine the reflection coefficient of each relay DTX- $r$ as follows:

$$
\left|\Gamma_{n, r}\right|^{2}=\max \left(0,1-\alpha_{0} / \alpha_{n, r}\right), \quad \forall r \in \mathcal{N}_{n} .
$$

If some DTx- $r$ has insufficient energy supply, i.e., $\alpha_{n, r}<\alpha_{0}$, we have $\left|\Gamma_{n, r}\right|=0$ and thus it will not participate in the passive relay communications. Note that the complex phase of $\Gamma_{n, r}$ can be aligned with the relay channel $g_{r, n} z_{n, r}$. Thus, we can reformulate the product of (11b) and (11c) as follows:

$$
e_{n, 1} o_{n}=\eta\left(\left|\mathcal{R}_{n}\right| \alpha_{0}+\beta_{n}\right)\left|g_{n, n}+\sum_{r \in \mathcal{R}_{n}} \Gamma_{r, n} g_{r, n} z_{n, r}\right|^{2},
$$

where $\left|\mathcal{R}_{n}\right|$ denotes the number of passive relays for DTX- $n$, i.e., $\left|\mathcal{R}_{n}\right|=\sum_{n \in \mathcal{N}_{n}} \mathbf{1}\left(\left|\Gamma_{n, r}\right|^{2}>0\right)$ and $\mathbf{1}(\cdot)$ is an indicator function. Note that $\left|\mathcal{R}_{n}\right|$ becomes smaller if the DUEs set a larger residual energy $\alpha_{0}$. Substituting (17) into (18), we find that the product $e_{n, 1} o_{n}$ in (18) is uniquely determined by the residual energy $\alpha_{0}$, given the scheduling and beamforming strategies $\left(\mathbf{t}, \mathbf{E}_{n, i}\right)$.

Till this point, we can sketch the ERE scheme by an iterative procedure as detailed in Algorithm 2. Firstly, we initialize the hybrid radio network, e.g., by the simple MRC scheme. With fixed scheduling and beamforming strategies $\left(\mathbf{t}, \mathbf{E}_{n, i}\right)$, we can evaluate the RF power $\alpha_{n, r}$ at each DTx- $n$ and correspondingly update the relay strategy by the rule in (17). One main step is to search for the optimal $\alpha_{0}$ that maximizes the objective in (11a). It is obvious that the maximum $\boldsymbol{\Lambda}\left(\alpha_{0}\right)$ depends on the choice of $\alpha_{0}$. Hence, the optimal $\alpha_{0}$ can be determined by one-dimension search, e.g., the bisection algorithm. Given the error bound $\epsilon$, the number of iterations required in the bisection search method will be $\log _{2}\left(\epsilon_{0} / \epsilon\right)$, where $\epsilon_{0}$ denotes the maximum value of $\alpha_{0}$ and can be simply set as the maximum of $\alpha_{n, r}$. With fixed $\alpha_{0}$, we can evaluate 


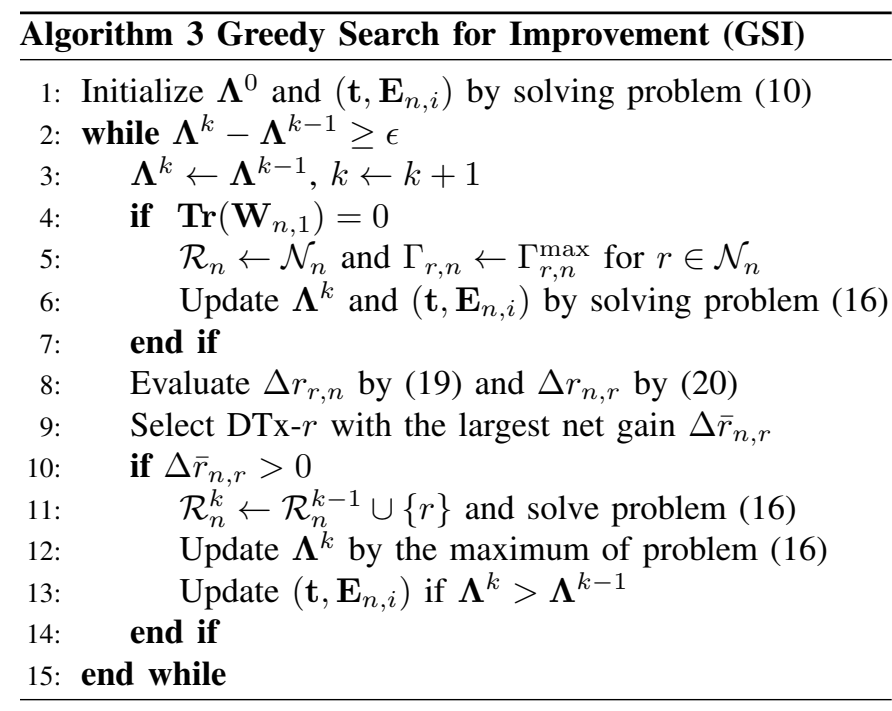

$\boldsymbol{\Lambda}\left(\alpha_{0}\right)$ efficiently by the following convex problem:

$$
\max _{\mathbf{t}, \mathbf{E}_{n, i}} \sum_{n \in \mathcal{N}}\left(\tilde{r}_{n}^{A}+r_{n}^{P}\right) \quad \text { s.t. (11d) and (18). }
$$

Once we find the optimal $\alpha_{0}$, we update the optimal reflection coefficients by (17). This will incur a new round of update to the scheduling and beamforming strategies $\left(\mathbf{t}, \mathbf{E}_{n, i}\right)$ by solving an SDP in the form of problem (16). Note that the convergence of Algorithm 2 is guaranteed as the iterative update of $\left(\mathbf{t}, \mathbf{E}_{n, i}\right)$ and $\Gamma_{n, r}$ follows an alternating optimization method.

The SRO algorithm provides an approximate reformulation to optimize the sum throughput performance. The MRC and ERE algorithms are based on simple heuristics with reduced computational complexities and good practical performance. Whereas all these algorithms provide approximate solutions to the original design problem. The performance gap with respect to its maximum is not known. In the following, we also provide the greedy search algorithm for performance improvement (GSI) to evaluate the maximum performance, which serves as a baseline for performance comparison.

3) Greedy Search for Improvement (GSI): The basic idea of the GSI scheme is to iteratively search for a better relay set $\mathcal{R}_{n}$ for each DTx- $n$ that can improve its performance gain. Given the relay set $\mathcal{R}_{n}$, any relay DTx- $r$ sets $\Gamma_{r, n}^{\max }$ to relay information for DTx- $n$. Considering a special case of problem (11), if there is not power allocation on the first subslot $t_{n, 1}$, i.e., $\operatorname{Tr}\left(\mathbf{W}_{n, 1}\right)=0$, any other DTx- $r$ for $r \neq n$ will decide to relay information for DTx- $n$ as this does not impair the energy harvesting opportunities of DTx-r. Hence, we can simply set $\Gamma_{r, n}$ to its maximum for $r \in \mathcal{N}_{n}$. For a more general case with $\operatorname{Tr}\left(\mathbf{W}_{n, 1}\right)>0$, the relay DTx- $r$ has to decide whether it is beneficial to perform passive relaying for DTx- $n$, as this will sacrifice its energy harvesting opportunity in the sub-slot $t_{n, 1}$.

To proceed, we initialize the system by the solution to problem (10) or (16) and obtain a lower bound $\boldsymbol{\Lambda}^{0}$ on the throughput performance. The update of relay set $\mathcal{R}_{n}$ depends on the losses and gains by evaluating different relay strategies. In particular, when DTx- $r$ decides to relay for DTx- $n$, its energy loss $\Delta e_{r, n}$ and throughput loss $\Delta r_{r, n}$ (due to its

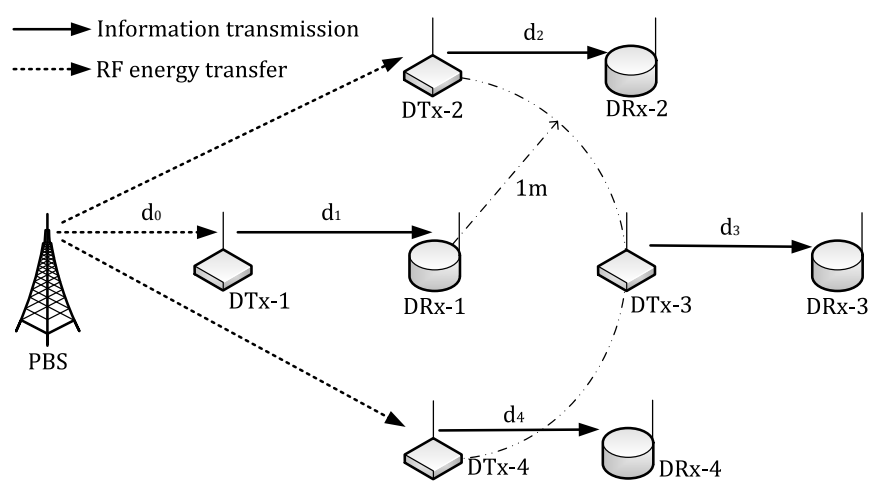

Fig. 3: The network configuration under evaluation.

assistance for DTx- $n$ ) can be characterized as follows:

$$
\begin{aligned}
\Delta e_{r, n} & =\eta \mathbf{h}_{r}^{H} \mathbf{E}_{n, 1} \mathbf{h}_{r}, \text { and } \\
\Delta r_{r, n} & =t_{r, 1} \log \left(\frac{1+e_{r, 1}\left|\tilde{g}_{n, n}\right|^{2} / t_{r, 1}}{1+\left(e_{r, 1}-\Delta e_{r, n}\right)\left|\tilde{g}_{n, n}\right|^{2} / t_{r, 1}}\right) .
\end{aligned}
$$

In another aspect, the channel of DTx- $n$ is enhanced as $\tilde{g}_{n, n}\left(\mathcal{R}_{n} \cup\{r\}\right)=\tilde{g}_{n, n}\left(\mathcal{R}_{n}\right)+\Gamma_{r, n} g_{r, n} z_{n, r}$. Then, the throughput gain of DTx-n (due to the assistance from DTx-r) can be evaluated as:

$$
\Delta r_{n, r}=t_{n, 1} \log \left(\frac{1+e_{n, 1}\left|\tilde{g}_{n, n}\left(\mathcal{R}_{n} \cup\{r\}\right)\right|^{2} / t_{n, 1}}{1+e_{n, 1}\left|\tilde{g}_{n, n}\left(\mathcal{R}_{n}\right)\right|^{2} / t_{n, 1}}\right) .
$$

The GSI scheme is detailed in Algorithm 3, in which we iteratively update the relay set $\mathcal{R}_{n}$ based on the evaluation of performance losses and gains. Let $\boldsymbol{\Lambda}^{k}$ be the best lower bound in the $k$-th iteration. In each time slot $t_{n}$, we calculate the net performance gain $\Delta \bar{r}_{n, r}=\Delta r_{n, r}-\Delta r_{r, n}$ for each relay DTx$r$, and then select DTx- $r$ with the largest gain as the candidate relay. If $\Delta \bar{r}_{n, r}>0$, we solve a convex problem in the form of problem (16) with the new relay set $\mathcal{R}_{n}^{k+1}=\mathcal{R}_{n}^{k} \cup\{r\}$. If the optimal throughput is greater than the current lower bound $\boldsymbol{\Lambda}^{k}$, we then update the relay set, the PBS's energy beamforming, and time allocation strategies.

\section{NUMERICAL EVALUATION}

In this part, we evaluate the throughput performance of different transmission strategies. Without loss of generality, we consider $M=3$ antennas at the PBS and its maximum transmit power (in watt) is set by $P_{\max }=0.2$. The energy budget (in Joule) in one data frame is given by $E_{\max }=0.4$ unless otherwise stated. The channel path loss exponent is set to 2 . The path loss measured at unit distance is $-30 \mathrm{~dB}$ and the antenna gain from the PBS to each user is $15 \mathrm{~dB}$. This can be achieved by using the directional Antenna AC-D24W08 with $12 \mathrm{dBi}$ antenna gain at the PBS and a commercial monopole antenna with $3 \mathrm{dBi}$ antenna gain at the IoT devices. The channel bandwidth is set to $100 \mathrm{kHz}$ and the ambient RF power density is assumed as $-100 \mathrm{dBm}$. The RF-DC energy conversion efficiency is $\eta=0.5$.

\section{A. Performance of SCA-based Relay Optimization}

We first evaluate the sum throughput by considering a fixed topology, as illustrated in Fig. 3. There are $N=4$ DUE 
transceiver pairs distributed under the coverage of the PBS, which provides both RF energy and carrier signals for the active and passive radios, respectively. The distance in meters from the PBS to the nearest transmitter, i.e., DTx-1, is set as $d_{0}=2$. The distances $d_{n}$ between each transceiver pair is set as $d_{n}=3$. In the SRO scheme, we consider the simplified case that all DUEs set the same reflection coefficient $\Gamma$. This can be easily extended to the general case with different reflection coefficients for individual relays. The performance dynamics in the SRO scheme is shown in Fig. 4(a), which verifies the convergence of sum throughput performance after a few number of iterations. It is obvious that the sum throughput increases in the energy budget $E_{\max }$. Fig. 4(b) shows the reflection coefficient in different iterations. When the energy budget $E_{\max }$ is limited, we observe a larger reflection coefficient which implies that the DUEs tend to cooperate with each other. In particular, the reflection coefficient goes up to unity after a few iterations as $E_{\max } \leq 0.15$. In this case, the SRO scheme degenerates to the simple MRC scheme. Different $E_{\max }$ also indicates the DUEs' preferences for cooperation, i.e., a smaller $E_{\max }$ urges the DUEs to converge faster to the unity value, as shown in Fig. 4(b).

In Fig. 5, we show the active transmission time $t_{n, 1}$ of different DTx- $n$ with the SRO scheme. Note that DTx-2 and DTx-4 are in symmetric positions. They experience similar channel conditions in expectation and thus achieve similar transmission scheduling strategies. As such, we only show the convergence results of $t_{n, 1}$ for $n \in\{1,2,3\}$ in Fig. 5(a). We set the energy budget as $E_{\max }=0.20$ and the initial values $t_{n, 1}^{0}$ by the solution to (10), which only allows for mode switch without DUEs' cooperation. Typically, the DUEs with better channel conditions can harvest more energy from the PBS, and thus tend to transmit more with the active radio mode. After a few iterations, each DUEs' active transmission time converges to a stable level and we have $t_{1,1}>t_{2,1}>t_{3,1}$. Note that the convergent value of $t_{1,1}$ is very close to its initial value, which implies that DTx-1 can harvest sufficient energy and the passive relaying scheme has little effect on its transmission strategy. While the active transmission time for DTx-2 and DTx-3 decreases significantly compared to the initial value. This implies that DTx-2 or DTx-3 prefers to passively relay for DTx-1, instead of transmitting information by itself. Such cooperation can improve the overall throughput performance, as demonstrated in Fig. 4(a).

In Fig. 5(b), we further show the impact of energy budget $E_{\max }$ on the DUEs' transmission scheduling strategies. We gradually increase $E_{\max }$ and evaluate the active transmission time $t_{n, 1}$ of each DTx- $n$. Though we always have $t_{1,1}>$ $t_{2,1}>t_{3,1}$, different DUE's active transmission time shows a very diverse shape as shown in Fig. 5(b). When energy budget is small, e.g., $E_{\max } \leq 0.10$, the DTx- 1 can always transmit in the active mode and the DTx- 3 can only operate in the passive mode due to insufficient energy harvesting opportunities. An interesting observation is that the active transmission time may drop as $E_{\max }$ increases, e.g., $t_{1,1}$ and $t_{2,1}$ decrease for $E_{\max } \leq 0.15$. In this case, the passive mode may outperform the active mode, and thus each DTx$n$ will split more time in the passive mode. When $E_{\max }$

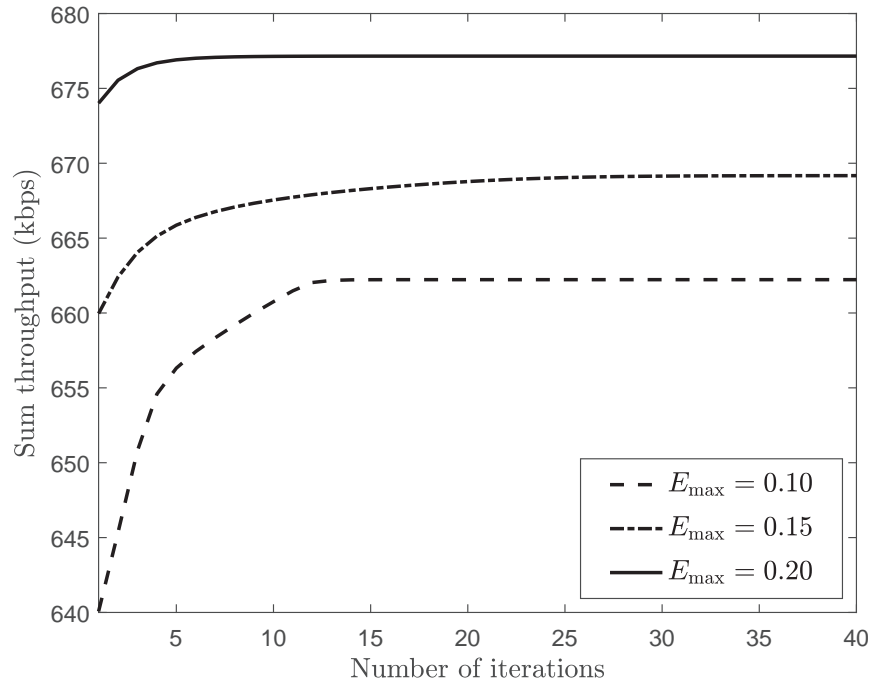

(a) Throughput dynamics in the SRO scheme.

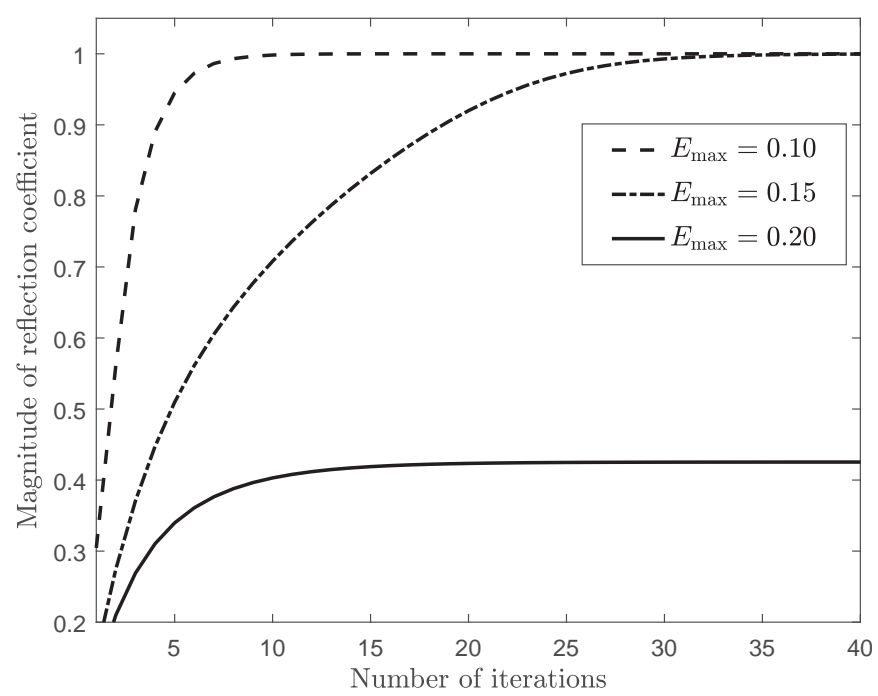

(b) Convergence of reflection coefficient.

Fig. 4: The iterations and convergence of the SRO scheme.

further increases, i.e., $E_{\max }>0.15$, the DUEs tend to harvest more energy and refrain from relaying for other users. Hence, we observe that the DUEs split more time in the active mode as verified in Fig. 5(b). We also compare the active transmission time between the SRO and the simple MRC scheme in Fig. 5(b). Note that the MRC scheme enforces full and myopic cooperation among all DUEs, which sacrifices individual DUEs' energy harvesting opportunities. This results in a decreased active transmission time in the MRC scheme, compared to that of the SRO scheme.

\section{B. Comparison of Different Passive Relaying Schemes}

In this part, we evaluate the average performance gain achievable by different passive relaying schemes. We assume $N=10$ transceiver pairs with randomly generated channels and distributed around the PBS in the experiments. We average the throughput performance for 100 simulation runs and show the comparison results in Fig. 6. The common observation is that the sum throughput of different strategies all increase in 


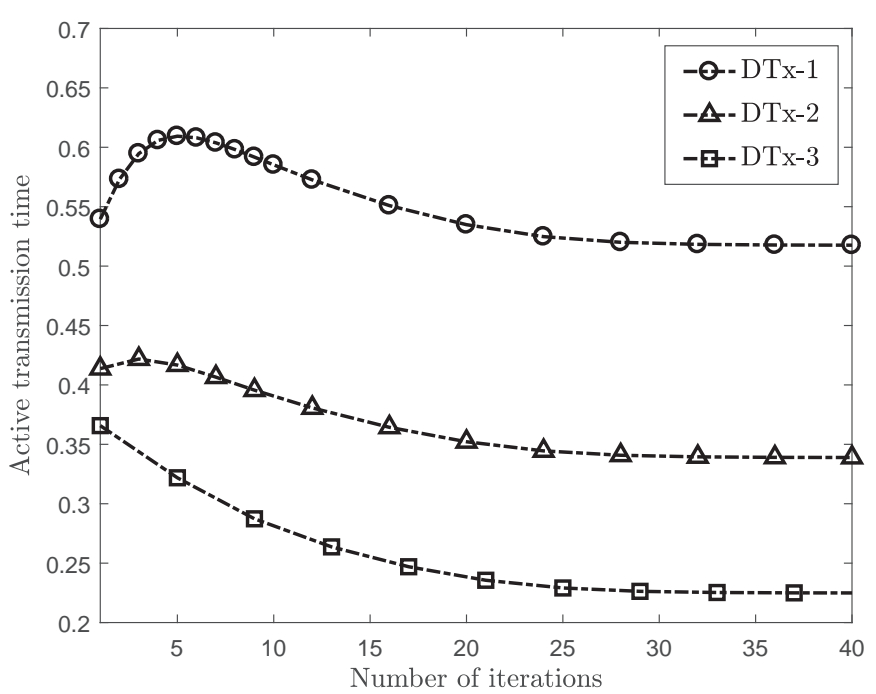

(a) Convergence of DUEs' active transmission time.

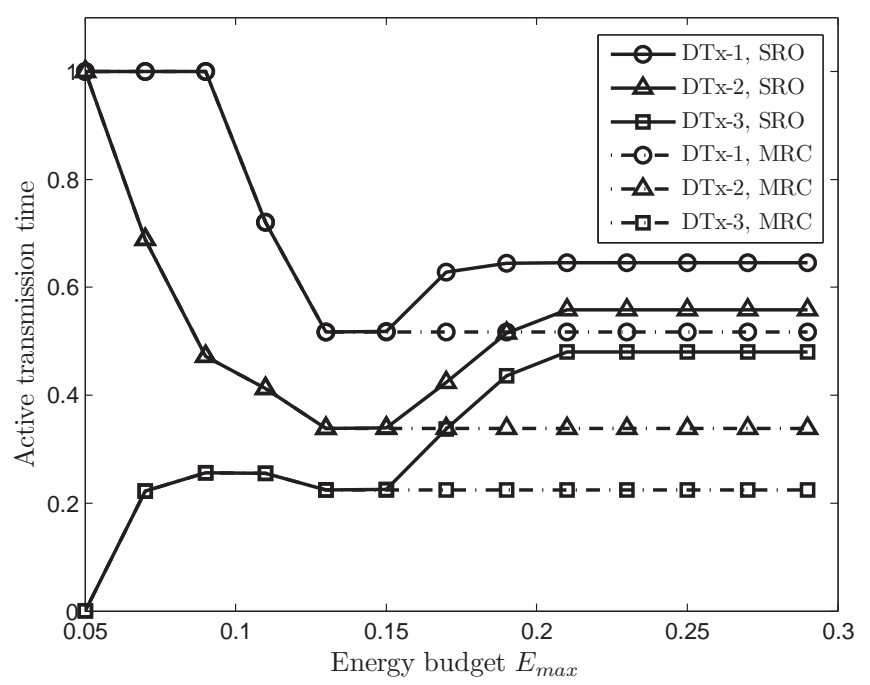

(b) Energy budget affects DUEs' active transmission time.

Fig. 5: Dynamics of the active transmission time.

terms of the PBS' energy budget $E_{\max }$. When $E_{\max }$ becomes high, the energy harvested by different DUE is bounded by the maximum transmit power $P_{\max }$. Hence, the sum throughput performance remains the same when $E_{\max } \geq 0.20$.

In Fig. 6(a), we show the performance gain of passive relaying schemes comparing to the non-cooperative cases. The All-Passive (or All-Active) scheme as shown in Fig. 6(a) assumes that all DUEs only operate in passive (or active) mode. The SWT scheme in Fig. 6(a) is set by the solution to (10), which only supports radio mode switching in the hybrid access model. Note that both the single-mode (i.e., All-Passive and All-Active) and the SWT schemes are non-cooperative cases. Firstly, we observe that the All-Active scheme performs much better than the All-Passive scheme in the proposed setting. The performance gain is stable and significant when energy budget $E_{\text {max }}$ is high. In this case, the DUEs can harvest sufficient energy and achieve better throughput performance in active mode. The SWT scheme further increases the throughput performance due to the flexible mode switching. Moreover,

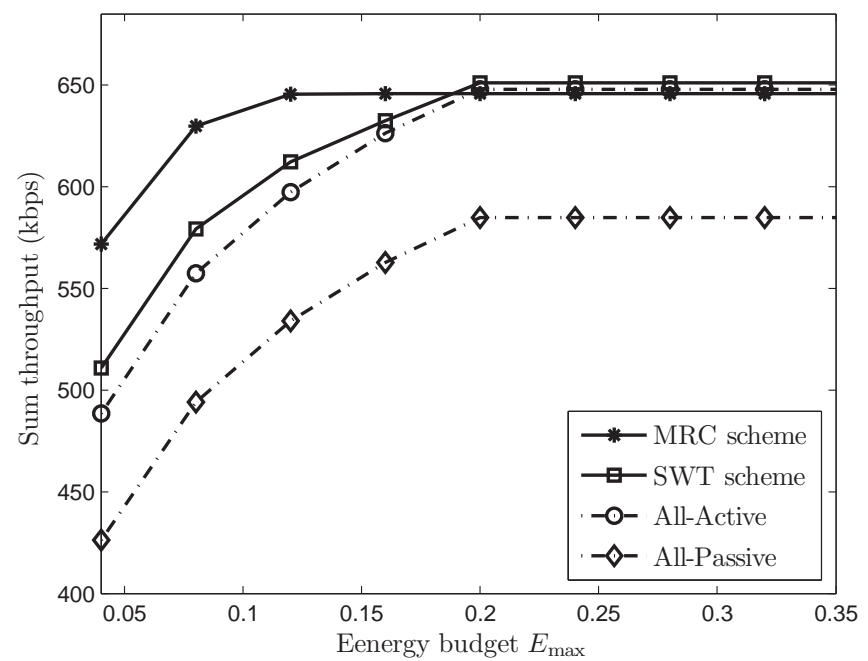

(a) Performance improvement over non-cooperative cases.

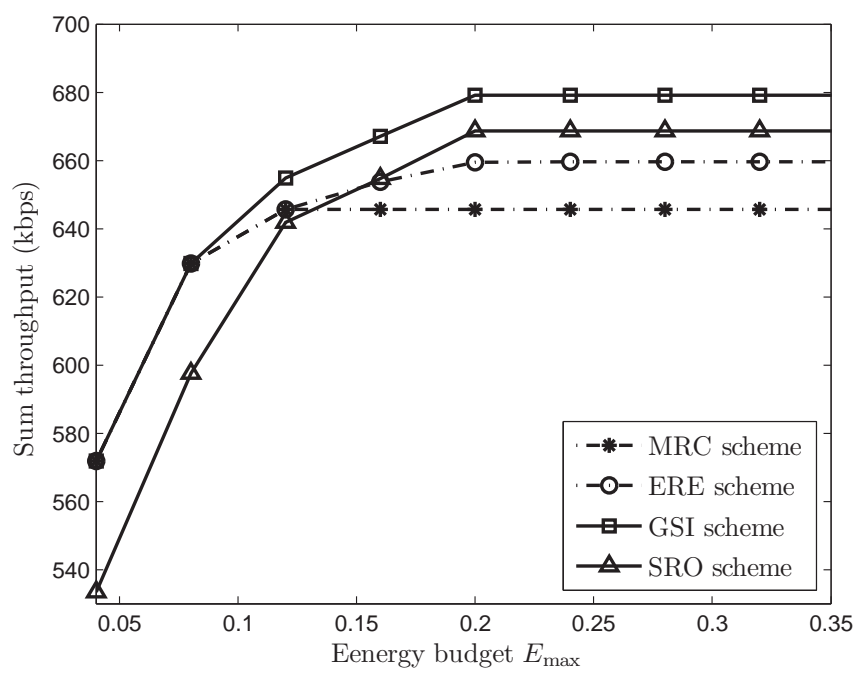

(b) Comparison of passive relaying schemes.

Fig. 6: The performance evaluation of passive relaying schemes.

we observe that the simple MRC scheme, by enforcing passive relaying among different DUEs, achieves significant improvement in the sum throughput compared to the non-cooperative cases, especially when $E_{\max }$ is small. We also note that the throughput is slightly decreased in the MRC scheme when $E_{\max }>0.2$. This implies that the DUEs prefer to operate independently when energy supply is sufficient.

In Fig. 6(b), we examine the throughput performance of different passive relaying schemes. It is obvious that the sum throughput of all schemes increases with $E_{\max }$. Besides, we observe that the GSI scheme achieves the maximum throughput performance. However, it demands frequent and extensive information exchange among DUEs to evaluate the loss and gain of each potential relay strategy. The iteration in Algorithm 3 also has slow convergence. These drawbacks make it difficult for practical implementation, whereas we can serve the GSI scheme as a benchmark for performance comparison. The SRO scheme provides the one-shot optimization of the relaying strategy. Though the SRO scheme is based on the 


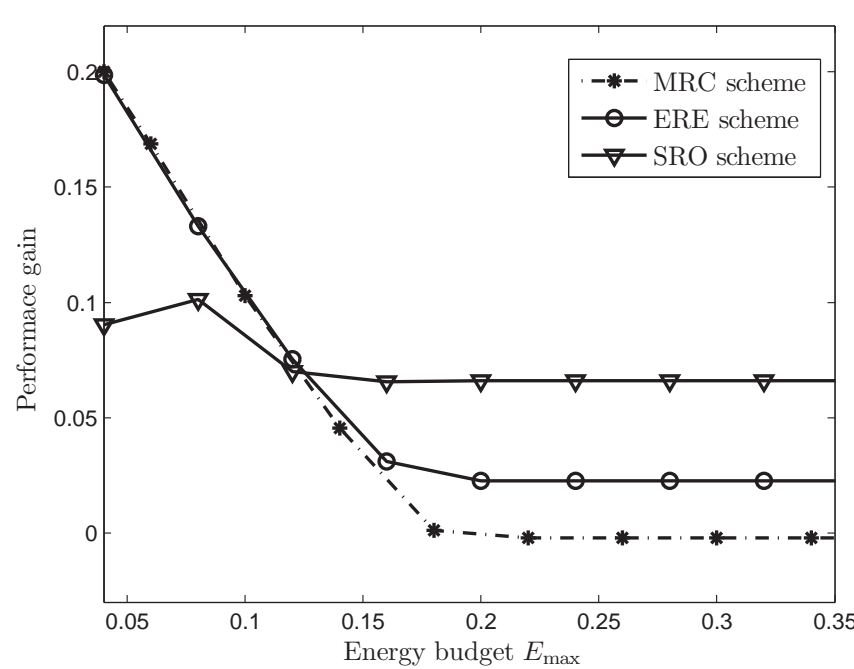

Fig. 7: Performance gain of different passive relaying schemes.

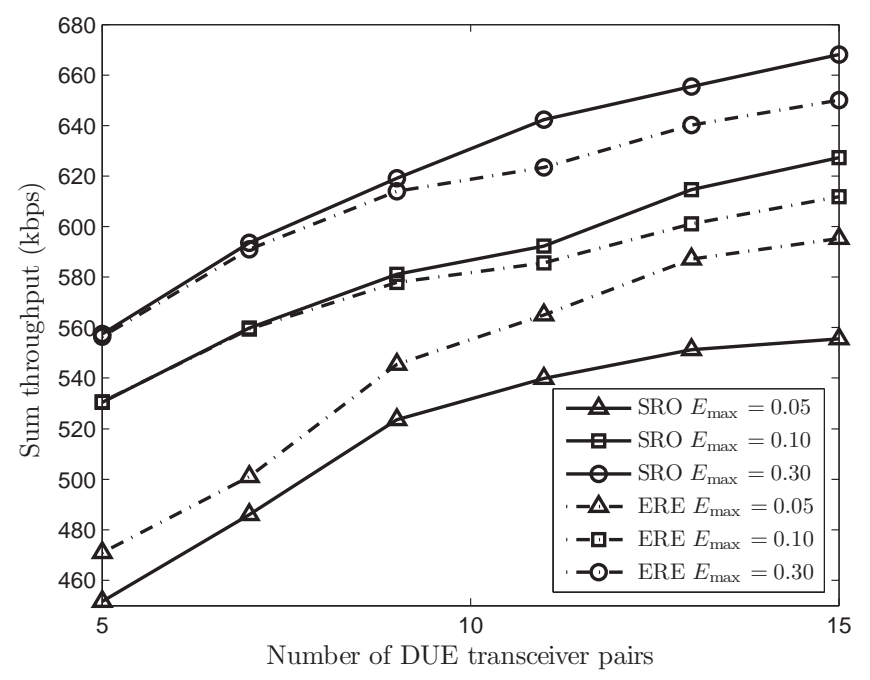

Fig. 8: Sum throughput increases with the node density.

SCA method, the iterations can be performed efficiently in a centralized manner, e.g., by the PBS or the edge controller as shown in Fig. 1(a). Thus, it can practically reduce the demand for information exchange and speed up the optimization of the relaying strategy. Moreover, the throughput performance of the SRO scheme is actually very close to that of the GSI scheme for a wide range of $E_{\max }$, as shown in Fig. 6(b). To further reduce the computational complexity of the SRO scheme, we further proposed the heuristic ERE scheme which achieves the optimal performance when $E_{\max }$ is low and significantly outperforms the MRC scheme when $E_{\max }$ becomes large. Most importantly, the ERE scheme can be implemented in a decentralized manner. Given the residual energy $\alpha_{0}$, each DUE can make relaying decisions based on its perception of the RF power. The computational complexity is also insignificant by calculating the reflection coefficient directly via (17).

\section{Performance Gain vs. Energy Budget and User Densities}

In Fig. 7, we show the performance gain of different relay schemes compared to the non-cooperative SWT scheme as a benchmark. The number of DUEs under the PBS' coverage is fixed at $N=15$. We observe that the MRC scheme achieves very close performance to the ERE scheme with small $E_{\max }$. In particular, it achieves $10 \%-20 \%$ performance gain compared to the SWT scheme. With small $E_{\max }$, there will be limited power allocation in the first sub-slots and thus the DUEs are motivated to relay for each other. When $E_{\max }$ increases, the MRC scheme becomes worse off which implies that the DUEs prefer to transmit by themselves. In this case, the SRO scheme becomes the optimal relay strategy and achieves $7 \%$ performance gain. The simulation result in Fig. 7 suggests an adaptive relaying scheme that adopts the simple MRC scheme when $E_{\max }$ is small and changes to the SRO or ERE scheme when $E_{\max }$ becomes large.

We image that the node density in D2D network also has significant impact on the throughput performance. A higher node density implies a shorter distance in average among different DUEs and more neighboring DUEs can be chosen as the candidates of passive relays. To verify this, we gradually increase the number of DUE transceiver pairs in the same network and evaluate the average throughput performance as shown in Fig. 8. It is obvious that the sum throughput of the SRO and ERE schemes all increase with the node density. This implies that the passive replying scheme can be more profitable in a dense D2D network, in which neighboring DUEs are more preferable to assist each other via backscatter communications. We also observe that $E_{\max }$ has different impacts on the SRO and the ERE schemes. In particular, the ERE scheme achieves very close performance as that of the SRO scheme when $E_{\max }$ is large. However, it significantly outperforms the SRO scheme when it becomes low, i.e., $E_{\max }=0.05$, and the performance gap increases with the node density. This result implies that the ERE scheme can be a better choice for practical implementation, i.e., it performs better with small $E_{\max }$ and achieves similar performance as that of the SRO scheme with large $E_{\max }$.

\section{CONCLUSIONS}

In this paper, we study a wireless powered D2D network, in which each transceiver can switch between active and passive radio modes. We formulate and solve a sum throughput maximization problem by jointly optimizing the energy beamforming strategy, the power control and transmission scheduling in two radio modes. To account for users' cooperation, we propose the passive relaying communications and reformulate the throughput maximization problem. Both approximate and heuristic algorithms are proposed to determine the optimal relaying strategy for the hybrid radio network. Simulation results reveal that a significant throughput enhancement can be achieved by exploiting the radio diversity gain and the user cooperation gain. 


\section{REFERENCES}

[1] X. Lu, D. Niyato, H. Jiang, D. I. Kim, Y. Xiao, and Z. Han, "Ambient backscatter assisted wireless powered communications," IEEE Wireless Commun., vol. 25, no. 2, pp. 170-177, Apr. 2018.

[2] X. Lu, P. Wang, D. Niyato, D. I. Kim, and Z. Han, "Wireless networks with rf energy harvesting: A contemporary survey," IEEE Commun. Surv. Tut., vol. 17, no. 2, pp. 757-789, 2015.

[3] S. Gong, J. Xu, L. Gao, X. Huang, and W. Liu, "Passive relaying scheme via backscatter communications in cooperative wireless networks," in proc. IEEE WCNC, Barcelona, Spain, Apr. 2018.

[4] W. Chen, C. Li, S. Gong, L. Gao, and J. Xu, "Joint transmission scheduling and power allocation in wirelessly powered hybrid radio networks," in proc. IEEE Int. Conf. Comput., Network. Commun. (IEEE ICNC), Honolulu, USA, Feb. 2019.

[5] D. Darsena, G. Gelli, and F. Verde, "Modeling and performance analysis of wireless networks with ambient backscatter devices," IEEE Trans. Commun., vol. 65, no. 4, pp. 1797-1814, Apr. 2017.

[6] W. Chen, W. Liu, L. Gao, S. Gong, C. Li, and K. Zhu, "Backscatteraided relay communications in wireless powered hybrid radio networks," in proc. IEEE WCNC (submitted), Marrakech, Morocco, Apr. 2019.

[7] C. Boyer and S. Roy, "Backscatter communication and RFID: Coding, energy, and MIMO analysis," IEEE Trans. Commun., vol. 62, no. 3, pp. 770-785, March 2014.

[8] B. Kellogg, V. Talla, S. Gollakota, and J. R. Smith, "Passive Wi-Fi: Bringing low power to Wi-Fi transmissions," in proc. 13th USENIX Symposium on Networked Systems Design and Implementation (NSDI 16), Santa Clara, CA, Mar. 2016, pp. 151-164.

[9] J. F. Ensworth and M. S. Reynolds, "BLE-Backscatter: Ultralow-power IoT nodes compatible with bluetooth 4.0 low energy (BLE) smartphones and tablets," IEEE Trans. Microw. Theory Techn., vol. 65, no. 9, pp. 3360-3368, Sep. 2017.

[10] V. Liu, A. Parks, V. Talla, S. Gollakota, D. Wetherall, and J. R. Smith, "Ambient backscatter: Wireless communication out of thin air," in Proc. ACM SIGGOMM, Aug. 2013.

[11] A. N. Parks, A. Liu, S. Gollakota, and J. R. Smith, "Turbocharging ambient backscatter communication," in Proc. ACM SIGGOMM, Aug. 2014.

[12] G. Yang and Y. C. Liang, "Backscatter communications over ambient OFDM signals: Transceiver design and performance analysis," in Proc. IEEE GLOBECOM, Dec. 2016, pp. 1-6.

[13] C. Yang, J. Gummeson, and A. Sample, "Riding the airways: Ultrawideband ambient backscatter via commercial broadcast systems," in proc. IEEE INFOCOM, Atlanta, GA, May 2017.

[14] B. Kellogg, V. Talla, S. Gollakota, and J. R. Smith, "Passive Wi-Fi: Bringing low power to Wi-Fi transmissions," in proc. 13th USENIX Symposium on Networked Systems Design and Implementation (NSDI 16), Mar. 2016, pp. 151-164.

[15] G. Wang, F. Gao, R. Fan, and C. Tellambura, "Ambient backscatter communication systems: Detection and performance analysis," IEEE Trans. Commun., vol. 64, no. 11, pp. 4836-4846, Nov. 2016.

[16] Q. Tao, C. Zhong, H. Lin, and Z. Zhang, "Symbol detection of ambient backscatter systems with manchester coding," IEEE Trans. Wireless Commun., vol. 17, no. 6, pp. 4028-4038, Jun. 2018.

[17] D. T. Hoang, D. Niyato, P. Wang, D. I. Kim, and Z. Han, "Ambient backscatter: A new approach to improve network performance for RFpowered cognitive radio networks," IEEE Trans. Commun., vol. 65, no. 9, pp. 3659-3674, Sep. 2017.

[18] G. Yang, D. Yuan, and Y. Liang, "Stackelberg game for distributed time scheduling in rf-powered backscatter cognitive radio networks," IEEE Trans. Wireless Commun., vol. 17, no. 8, 2018.

[19] _ - "Optimal time scheduling for wireless-powered backscatter communication networks," IEEE Wireless Commun. Lett., vol. 7, no. 5, pp. 820-823, 2018.

[20] Q. Yang, H. Wang, T. Zheng, Z. Han, and M. H. Lee, "Wireless powered asynchronous backscatter networks with sporadic short packets: Performance analysis and optimization," IEEE Internet of Things Journal, vol. 5, no. 2, pp. 984-997, Apr. 2018.

[21] G. Yang, D. Yuan, and Y. Liang, "Throughput maximization for hybrid backscatter assisted cognitive wireless powered radio networks," IEEE IoT Journal, vol. 5, no. 3, pp. 2015-2024, Jun. 2018.

[22] J. Li, J. Xu, S. Gong, C. Li, and D. Niyato, "A game theoretic approach for Backscatter-Aided relay communications in hybrid radio networks," in proc. IEEE GLOBECOM, Abu Dhabi, United Arab Emirates, Dec. 2018.
[23] G. Yang, D. Yuan, and Y. Liang, "User cooperation in wireless-powered backscatter communication networks," IEEE Wireless Commun. Lett., vol. 5, no. 3, pp. 2015-2024, Jun. 2018.

[24] _ - "Optimal time allocation in relay assisted backscatter communication systems," in proc. IEEE VTC Spring, 2018.

[25] _ , "Performance analysis of wireless-powered relaying with ambient backscatter," in proc. IEEE ICC, 2018.

[26] F. Gao, R. Zhang, and Y.-C. Liang, "Channel estimation for OFDM modulated two-way relay networks," IEEE Trans. Signal Proces., vol. 57, no. 11, pp. 4443-4455, Nov. 2009.

[27] S. Gong, X. Huang, J. Xu, W. Liu, P. Wang, and D. Niyato, "Backscatter relay communications powered by wireless energy beamforming," IEEE Trans. Commun., vol. 66, no. 7, pp. 3187-3200, Feb. 2018.

[28] R. Long, H. Guo, G. Yang, Y. Liang, and R. Zhang, "Symbiotic radio: A new communication paradigm for passive internet-of-things," CoRR, vol. abs/1810.13068, 2018.

[29] D. Li, W. Peng, and Y. Liang, "Hybrid ambient backscatter communication systems with harvest-then-transmit protocols," IEEE Access, vol. 6, pp. $45288-45298,2018$.

[30] B. Smida and S. Khaledian, "Reflectfx: In-band full-duplex wireless communication by means of reflected power," IEEE Transactions on Communications, vol. 65, no. 5, pp. 2207-2219, May 2017.

[31] E. Boshkovska, D. W. K. Ng, N. Zlatanov, and R. Schober, "Practical non-linear energy harvesting model and resource allocation for SWIPT systems," IEEE Commun. Lett., vol. 19, no. 12, pp. 2082-2085, 2015.

[32] G. Yang, D. Yuan, and Y. Liang, "Optimal resource allocation in fullduplex ambient backscatter communication networks for green iot," CoRR, vol. abs/1805.01365, 2018.

[33] M. Lee, G. Yu, and G. Y. Li, "Learning to branch: Accelerating resource allocation in wireless networks," CoRR, vol. abs/1903.01819, 2019.

[34] M. Grant and S. Boyd, "CVX: Matlab software for disciplined convex programming, version 2.1," http://cvxr.com/cvx, Mar. 2014.

[35] Y. Nesterov and A. Nemirovskii, Interior-Point Polynomial Algorithms in Convex Programming. Society for Industrial and Applied Mathematics, 1994.

[36] J. Li, J. Xu, S. Gong, X. Huang, and P. Wang, "Robust radio mode selection in wirelessly powered communications with uncertain channel information," in Proc. IEEE GLOBECOM, Dec. 2017.

[37] S. Gong, X. Huang, J. Xu, W. Liu, P. Wang, and D. Niyato, "Backscatter relay communications powered by wireless energy beamforming," IEEE Transactions on Communications, vol. 66, no. 7, pp. 3187-3200, Jul. 2018.

[38] Z.-Q. Luo, W.-K. Ma, A.-C. So, Y. Ye, and S. Zhang, "Semidefinite relaxation of quadratic optimization problems," IEEE Signal Proces. Mag., vol. 27, no. 3, pp. 20-34, May 2010. 